\title{
PIM1-dependent phosphorylation of Histone H3 at Serine 10 is required for MYC-dependent transcriptional activation and oncogenic transformation.
}

Alessio Zippo, Alessandra De Robertis, Riccardo Serafini, and Salvatore Oliviero

Dipartimento di Biologia Molecolare Universita’ di Siena

Via Fiorentina 1, 53100 Siena, Italy.

Running title: PIM1 phosphorylates H3S10

Key words: transcriptional regulation, MYC, PIM1, chromatin, nucleosome phosphorylation.

Correspondig Author :

Salvatore Oliviero

Dipartimento di Biologia Molecolare Universita' di Siena

Via Fiorentina 1, 53100 Siena, Italy.

e mail oliviero@unisi.it 
The serine/threonine kinase PIM1, cooperates with MYC in cell cycle progression and tumorigenesis. However, the nature of this cooperation remains elusive. Here we show that PIM1 contributes to the transcriptional activation of MYC-target genes by phosphorylating the histone H3 nucleosome at Serine 10 (H3S10). Recombinant PIM1 directly phosphorylates H3S10 on the nucleosome in vitro. Following growth factor stimulation, PIM1 accumulates in the nucleus where it forms a complex with the MYC/MAX dimer via the MYC BoxII domain (MBII). Immunofluorescence analysis coupled with in vivo run-on shows a high degree of PIM1 and MYC co-localization in the nucleus at sites of active transcription. Expression profile analysis revealed that PIM1 contributes to the regulation of $20 \%$ of the MYC-regulated genes. Chromatin immunoprecipitation (ChIP) analysis in MYC silenced cells demonstrates that MYC recruits PIM1 to the E boxes of the MYC-target genes FOSL1 (FRA1) and ID2. PIM1 knockdown as well as the over-expression of the kinase inactive mutant demonstrate that PIM1 is required for H3S10 phosphorylation at FOSL1 and ID2 MYC-binding sites and for their transcriptional activation. Moreover, we show that PIM1-dependent H3S10 phosphorylation contributes to MYC transforming capacity. These results establish a new function for PIM1 as a MYC cofactor that phosphorylates the chromatin at MYCtarget loci and suggest that nucleosome phosphorylation, at $\mathrm{E}$ boxes, contributes to MYC-dependent transcriptional activation and cellular transformation.

Chromatin structure plays a crucial role in eukaryotic gene transcription. Several transcription activators have been shown to recruit chromatin-modifying activities, which either mediate histone covalent modifications or ATP-dependent nucleosome mobilization. Covalent histone 
modifications including acetylation, methylation, phosphorylation and ubiquitinylation have been associated with gene regulation ${ }^{1,2}$. Phosphorylation of $\mathrm{H} 3 \mathrm{~S} 10$ has been connected with transcriptional activation in different organisms and with chromosome condensation during mitosis ${ }^{3,4}$. In mammalian cells, growth factor stimulation induces rapid phosphorylation of histone $\mathrm{H} 3$ at Serine 10 (H3S10) at c-Jun and c-Fos promoters mediated by MSK1, MSK2, and RSK2 $_{\text {kinases }}^{4-7}$. In response to inflammatory cytokines, IKK-ọphosphorylates H3S10 at NFkB-responsive promoters ${ }^{8,9}$.

In this study, we analyzed the function of PIM1, a constitutively active serine/threonine kinase. Pim1 was first identified as a common insertion site in $\mathrm{T}$ cell lymphomas induced by Molony murine leukemia virus $(\mathrm{Mo}-\mathrm{MuLV})^{10}$. RNA interference (RNAi) experiments demonstrated that human Pim1 (hereinafter referred to as PIM1) is essential for cell cycle progression of human umbilical vein endothelial cells (HUVEC) and for differentiation of endothelial precursors in vitro ${ }^{11}$. Myc and Pim1 have been shown to co-operate in cell growth and transformation. Pim1 over-expression in transgenic mice demonstrated its cooperation with cMyc in cell transformation ${ }^{12-15}$ and microarray expression profiling showed that Pim1 is overexpressed in Myc-driven prostate tumours ${ }^{16}$. However, the nature of c-Myc and Pim1 cooperation in cell transformation remains elusive.

Human c-Myc (hereinafter referred as MYC) codes for a basic helix-loop-helix leucine zipper (B-HLH) transcription factor that binds to the E box (CACGTG) when dimerized with MAX and regulates the transcription of distinct genes involved in cell cycle progression, apoptosis, cell growth, and differentiation ${ }^{17-23}$. The DNA binding domain of MYC is at the C-terminus while the N-terminal domain through the MYC boxII (MBII), essential for MYC-dependent 
cell transformation, has been shown to recruit multiprotein enzymatic complexes to the MYCactivated genes ${ }^{24}$.

To understand the function of PIM1, we investigated the molecular connection between PIM1 and MYC. We propose that the recruitment of PIM1 to the chromatin by MYC contributes to transcriptional activation of MYC-target genes by phosphorylating $\mathrm{H} 3 \mathrm{~S} 10$ at the $\mathrm{E}$ box element and suggest that this cooperation is relevant for MYC-dependent tumour formation.

\section{RESULTS}

\section{PIM1 phosphorylates the nucleosome at H3S10.}

PIM1 mRNA is induced with fast kinetics in HUVEC treated with VEGF-A ${ }^{11}$. Upon VEGF-A treatment PIM1 is induced and localizes in HUVEC nuclei within 40 minutes (see Supplementary Information Fig. S1). As PIM1 is a serine/threonine kinase with putative consensus sites present on histones, its nuclear localization could account for the increase of H3 phosphorylation observed in these cells between 60 and 90 minutes after VEGF-A treatment (see Supplementary Information Fig. S1). We found that PIM1 could directly phosphorylate nucleosomes in vitro by incubating the recombinant GST-PIM1 protein with chromatin fractions obtained from 293 cells in the presence of $\left[\tilde{\gamma}^{32} \mathrm{P}\right]$ ATP. This analysis revealed that a single protein, with molecular weight corresponding to $\mathrm{H} 3$, was phosphorylated by wild type GST-PIM1, but not by the kinase inactive mutant GST-PIM1-K67M (Fig. 1a, lanes 1-3). Specific inhibition of nucleosome phosphorylation was observed in the presence of a peptide derived from the $\mathrm{H} 3$ N-terminus but not with a peptide derived from $\mathrm{H} 2 \mathrm{~B}$ Nterminus or with an unrelated peptide (Fig. 1a, lanes 4-12). PIM1 could phosphorylate 
reconstituted nucleosomes containing either recombinant wild type $\mathrm{H} 3$ or $\mathrm{H} 3 \mathrm{~S} 28 \mathrm{~A}$, but not nucleosomes assembled with the H3S10A mutant or the double mutant (Fig. 1b) demonstrating that PIM1 phosphorylates $\mathrm{H} 3$ at S10. Histone $\mathrm{H} 3$ co-immunoprecipitated with transiently expressed PIM1 or the kinase inactive mutant suggesting that PIM1 associates in vivo with the nucleosome (Fig. 1c). Incubation of the immunoprecipitate in the presence of $\left[\gamma^{32} \mathrm{P}\right]$ ATP showed that the wild type PIM1, but not the kinase inactive mutant PIM1-K67M phosphorylated H3. We conclude that PIM1 associates in vitro and in vivo with $\mathrm{H} 3$ and directly phosphorylates $\mathrm{H} 3 \mathrm{~S} 10$ on the nucleosome.

\section{PIM1 forms a complex with MYC and MAX.}

VEGF-A treatment induces MYC and PIM1 co-localization in the cell nuclei at 60 minutes, decreasing thereafter (Fig. 2a). We tested whether PIM1 coimmunoprecipitaes with MYC. Time course analysis in HUVEC treated with VEGF-A showed that upon induction, both MYC and PIM1 were induced with fast kinetics. Immunoprecipitations with anti-MAX antibodies at various time points showed the formation of the MAX/MYC complex from 60 minutes after growth factor stimulation thus corresponding to the appearance of MYC in the nucleus (Fig. 2b, right panel). Importantly, PIM1 co-immunoprecipitated with MYC and MAX suggesting that a complex containing PIM1 is formed. Endogenous PIM1 was also found to co-immunoprecipitate with endogenous MYC and MAX from 293 cells after serum treatment for 120 minutes (Fig. 2c), thus demonstrating that a MAX/MYC/PIM1 endogenous complex is also formed in these cells.

As MYC-dependent cell transformation requires MYC boxII (MBII) domain to bind cofactors either in a TRRAP-dependent or -independent manner ${ }^{24}$, we tested whether PIM1 interacts 
with MYC at MBII via TRRAP. Wild type FLAG-MYC or MYC deleted in the MBII domain (FLAG-MYC MBII) were co-expressed with PIM1 in 293 cells and immunoprecipitated either with FLAG or PIM1 antibodies. Western blot analysis showed reciprocal immunoprecipitation of FLAG-MYC with PIM1, while co-immunoprecipitation was strongly reduced between FLAG-MYC MBII and PIM1 suggesting that MBII domain of MYC is required for the formation of a complex with PIM1 (Fig. 2d). Endogenous TRRAP associated with MYC but not with the FLAG-MYC MBII as previously described ${ }^{25}$. In contrast, PIM1 did not co-immunoprecipitate the endogenous TRRAP (lanes 5 and 10) suggesting that MYC forms independent complexes with PIM1 or TRRAP via the MBII domain.

\section{PIM1 co-localizes with MYC on the chromatin at actively transcribing loci.}

To measure PIM1 and MYC association at MYC-target genes, we performed a nuclear distribution analysis of PIM1 with respect to MYC, nascent transcripts, and chromatin markers. Quantitative analysis of double staining with anti-PIM1 and antibodies against different nuclear markers by sequential laser scanning (Fig. 3) showed an elevated degree of PIM1 colocalization with MYC with a Pearson's correlation coefficient $(\mathrm{Rr})$ of $0.82 \pm 0.06$. PIM1 also showed an elevated overlap with H3S10ph $(\operatorname{Rr} 0.85 \pm 0.07)$ and H3S10phK14ac while little colocalization could be detected between PIM1 and H3K9me2 (Rr 0.37 \pm 0.03$)$, a marker of silent heterochromatin. Furthermore, we analyzed the nuclear distribution of PIM1 with respect to active transcription sites by in situ transcriptional run-on experiments. Double labelling revealed a high degree of overlap between PIM1 and sites of active transcription $(\mathrm{Rr}$ $0.77 \pm 0.05)$ with a pattern resembling that of transcription factories ${ }^{26}$. A similar pattern of nuclear localization with respect to nascent transcripts was observed for MYC $(\operatorname{Rr} 0.81 \pm 0.09)$. 
Taken together these results suggest that following growth factor treatment PIM1 kinase is mostly localized with the transcription factor MYC at actively transcribing sites and with phosphorylated histones.

\section{H3S10 phosphorylation at the FOSL1 enhancer correlates with PIM1 association.}

Next we analysed PIM1 association and H3S10 phosphorylation at FOSL1 (also known as FRA1), a well characterized MYC-target gene ${ }^{27,28}$. FOSL1 contains an upstream functional SRE, which is not sufficient to confer promoter activation, but requires the activity of a downstream enhancer that is activated by MYC and AP- ${ }^{27-29}$. Upon VEGF-A treatment of HUVEC FOSL1 expression is induced with a peak at 60 minutes (Fig. 4a). To demonstrate a direct binding of MYC and PIM1 to the FOSL1 regulatory sequences ChIP followed by realtime quantitative PCR analysis was performed at various timepoints after VEGF-A treatment. As shown in Figure 4b, we generated primers to analyze four FOSL1 regions: a FOSL1 upstream region containing a functional SRE and a non canonical E box element ${ }^{30,31}$ (probe A), the downstream enhancer containing a canonical E box (probe B), a downstream region within the first intron (probe C), and a FOSL1 region within the second intron (probe D). Quantitative ChIP analysis with probe B revealed the transient recruitment to the FOSL1 enhancer of MYC and PIM1 from 60 minutes concomitant with a marked increase of H3S10ph signal (Fig. 4c). ChIP with probe B also showed a significant increase of H3S10phK9ac and H3S10phK14ac while H3K9acK14ac showed elevated levels before induction suggesting that, at the FOSL1 enhancer, $\mathrm{H} 3$ acetylation precedes MYC binding and $\mathrm{H} 3 \mathrm{~S} 10$ phosphorylation takes place at the acetylated $\mathrm{H} 3$.

Analysis of the upstream element (probe A) revealed MYC association with this DNA 
fragment. PIM1 did not associate with this element suggesting that the FOSL1 promoter is bound by an alternative MYC complex. This FOSL1 upstream element showed an increase of H3S10 phosphorylation at 15 minutes after VEGF-A treatment, which was therefore not dependent on PIM1. As the FOSL1 SRE element is constitutively occupied by the ternary complex factor (TCF), which is activated by the RAS/ERK pathway ${ }^{30}$, we further analyzed FOSL1 transcription and $\mathrm{H} 3 \mathrm{~S} 10$ phosphorylation in the presence of $\mathrm{H} 89$, a specific MSK1/MSK2 inhibitor ${ }^{5}$. Cell treatment with H89 affected FOSL1 transcription although a VEGF-A-dependent induction was still observed (Fig. 4d). The analysis of H3S10ph signal showed specific inhibition of H3S10 phosphorylation at the FOSL1 upstream element but not at the enhancer (Fig. 4e) demonstrating that phosphorylation at the upstream element and at the downstream enhancer are independent from each other and mediated by different kinases. The acetylation pattern at the FOSL1 upstream element showed a significant increase of $\mathrm{H} 3$ phosphorylation and acetylation with a peak between 15 and 30 minutes suggesting that, at this site, $\mathrm{H} 3 \mathrm{~S} 10$ phosphorylation precedes or is concomitant with $\mathrm{H} 3$ acetylation at $\mathrm{K} 9$ and $\mathrm{K} 14$ as previously described for IE promoters ${ }^{3,4}$.

The downstream FOSL1 fragments (analyzed with probes C and D) did not reveal MYC or PIM1 binding, nor the increase of phosphorylated H3S10, while presented elevated levels of acetylated H3. These results demonstrate specific recruitment of PIM1 to the FOSL1 enhancer concomitant with an increase of $\mathrm{H} 3 \mathrm{~S} 10$ phosphorylation at this site and with transcriptional activation of the gene.

\section{H3S10 phosphorylation at FOSL1 enhancer is mediated by PIM1.}

PIM1 depletion using small hairpin RNA (shRNA) was peformed to test whether PIM1 
association with the FOSL1 enhancer was required for H3S10 phosporylation. In 293 cells treated with serum MYC, PIM1, and FOSL1 showed a slower kinetics of induction with respect to HUVEC treated with VEGF-A possibly due to the different cell types (cell line versus primary cells). PIM1 silencing strongly inhibited FOSL1 mRNA expression (Fig. 7b and see Supplementary Information Fig. S3). Quantitative ChIP analysis (Fig. 5b and see Supplementary Information Fig. S2) demonstrated a peak of H3S10 phosphorylation at the FOSL1 enhancer (probe B as described in Fig. 4b) at 120 minutes that was significantly reduced by PIM1 silencing, while ChIP analysis at the FOSL1 upstream element (probe A) showed a faster kinetics of H3S10 phosphorylation that was not affected by PIM1 knockdown. No H3S10 phosphorylation was observed at the downstream fragments (probes $\mathrm{C}$ and $\mathrm{D}$ ). We concluded that H3S10 phosphorylation at the FOSL1 enhancer is PIM1-dependent.

PIM1 and MYC are found to associate at the FOSL1 enhancer with a peak between 90 and 120 minutes. PIM1 silencing significantly reduced PIM1 association at the enhancer without affecting MYC association. The overall pattern of $\mathrm{H} 3$ phospho-acetylation at $\mathrm{K} 9$ and $\mathrm{K} 14$ in 293 cells showed elevated levels of $\mathrm{H} 3$ acetylation already present before $\mathrm{H} 3$ phosphorylation at the FOSL1 enhancer (probe B) and along the FOSL1 gene (probe C and D) which was not affected by PIM1 silencing.

Moreover, expressing the kinase inactive mutant PIM1-K67M, significantly reduced H3S10ph levels at the FOSL1 enhancer (see Supplementary Information Fig. S4) demonstrating that the kinase activity of PIM1 is required for H3S10 phosphorylation at this site.

\section{PIM1 is recruited by MYC at the FOSL1 and ID2 E box elements.}

MYC silencing was performed to analyze whether its expression is required for PIM1 
recruitment to the FOSL1 enhancer (Fig. 6a and see Supplementary Information Fig. S5). We also analyzed MYC-dependent recruitment of PIM1 at the ID2 E box. ID2 is a well characterized MYC-regulated gene that, differently from FOSL1, contains its functional MYCbinding sites upstream the promoter ${ }^{28,32}$. As expected, ChIP analysis on control cells showed MYC association at the FOSL1 enhancer and the ID2 E box at 120 minutes after serum treatment, while MYC silencing significantly reduced MYC association at these sites (Fig. 6b and see Supplementary Information Fig. S5). ChIP analysis revealed PIM1 association with both genes in control cells while MYC knockdown significantly reduced PIM1 association at these E boxes (Fig. 6c and see Supplementary Information Fig. S5). Moreover, H3S10 phosphorylation showed significant reduction in MYC silenced cells at these sites (Fig. 6d and see Supplementary Information Fig. S5). In contrast, H3K9acK14ac was unaffected by MYC silencing (Fig. 6e and see Supplementary Information Fig. S5) confirming that H3 acetylation is not dependent on MYC binding. Importantly, the reintroduction of wild type FLAG-MYC, but not the FLAG-MYC MBII in MYC-silenced cells restored PIM1 recruitment and H3S10 phosphorylation at both sites (Fig. 6 f, g, h and see Supplemenrary Information Fig. S5). These results establish that MYC, via its MBII domain, recruits PIM1 to the E box elements of these MYC-regulated genes and its recruitment is required for phosphorylating H3S10 at these sites.

\section{PIM1 is required for transcriptional activation of FOSL1 and ID2 genes.}

After serum treatment of 293 cells, both FOSL1 and ID2 mRNA were induced with similar kinetics (Fig. 7b, e) corresponding to the peak of PIM1 and MYC association and H3S10 phosphorylation in these cells. The same results were obtained by measuring FOSL1 and ID2 unprocessed heterogeneous nuclear RNA (hnRNA) indicating that increased mRNA level 
depend on transcriptional activation. PIM1 silencing, as well as the expression of PIM1-K67M, inhibited induction of both FOSL1 and ID2 transcripts (Fig 7b, e and see Supplementary Information Fig. S6) demonstrating that PIM1 kinase activity is required for full transcriptional activation of both genes.

Quantitative ChIP analysis of RNA Polymerase II (Pol II) and as well as Pol II phosphorylated in Ser2 (Ser2P) at the C-terminal domain (CTD) of control cells revealed a significant increase in signal at FOSL1 and ID2 promoters and along the genes in response to serum treatment (Fig. 7c, f and see Supplementary Information Fig. S6) mirroring of the increase in transcript levels. PIM1 silencing led to a specific reduction of Pol II signal at the promoters and within the coding regions. This reduction, however, could not account for the inhibition of the transcriptional activity mediated by the PIM1 silencing. By contrast, the reduction of the elongating Pol II (Pol II-Ser2P) along the gene more closely correlated with transcript levels of both FOSL1 and ID2 suggesting that PIM1 silencing mostly affects the presence of Pol II engaged in transcript elongation.

\section{PIM1 contributes to the regulation of a subgroup of MYC-regulated genes.}

To understand the extent of PIM1 and MYC co-operation in gene transcription we performed expression profile analysis of 293 cells silenced either for MYC or PIM1 at 120 minutes after serum treatment. MYC silencing affected the expression of 1026 genes of which 818 were upregulated and 208 were down-regulated (see Supplementary Information Table SI) in line with previous analysis of MYC-regulated genes ${ }^{33}$. Comparison of genes regulated by MYC with those regulated by PIM1 showed that PIM1 contributes to the regulation of 207 genes out of the 1026 MYC-regulated genes. Thus, 20\% of MYC-regulated genes, are also regulated by 
PIM1. Functional characterization of these genes, based on Gene Onthology categorization through the Panther database (http://www.pantherdb.org), revealed that nucleic acid metabolism, transcriptional regulation, RNA processing, and oncogenesis are statistically overrepresented $(\mathrm{P}<0.05)$ (see Supplementary Information Table SII, SIII). Based on this analysis we conclude that PIM1 act as a cofactor for transcriptional regulation in a significant subset of MYC-dependent genes.

\section{PIM1 recruitment to the chromatin by MYC contributes to cell transformation.}

Next we investigated the biological relevance of MYC - PIM1 co-operation. First we verified whether PIM1 contributes to MYC-dependent regulation of cell cycle progression by silencing PIM1 in P493-6 cells, a cell line that depends on MYC expression for its proliferation ${ }^{27,34,35}$. Incorporation of $\left[{ }^{3} \mathrm{H}\right]$ thymidine was significantly decreased in PIM1 silenced cells and analysis of the cell cycle by FACS showed that PIM1 depletion determines a delay in S phase progression of these cells (see Supplementary Information Fig. S7).

To test whether PIM1 cooperation with MYC in cell transformation and tumour growth is due to PIM1's role in transcriptional activation of MYC-target genes, we performed transformation experiments in Rat-1 fibroblasts. Over-expression of MYC transforms these cells morphologically and induces them to grow in soft $\operatorname{agar}^{36}$. PIM1 silencing strongly inhibited the formation of soft agar foci, induced by MYC over-expression, and MYC silencing affected the number of foci induced by PIM1 over-expression to a similar extent, (Fig. 8a) thus, demonstrating that PIM1 is indeed required for MYC-dependent cell transformation. Moreover, the over-expression of MYC and PIM1 generates a significantly higher number of soft agar colonies than cells over-expressing either MYC or PIM1 (Fig. 8a, b). 
To understand whether PIM1 acts as a MYC cofactor in cell transformation we generated chimeric constructs in which PIM1 was directly fused to the $\mathrm{N}$ terminal domain of MYC (PIM1-MYC). We found that the PIM1-MYC fusion molecule induced a comparable number of soft agar colonies relative to the co-expression of MYC and PIM1 in the same cells (Fig. 8b) suggesting that PIM1 recruitment to the chromatin by $\mathrm{MYC}$ is required for cooperation between MYC and PIM1 in cell transformation. Since formation of the MYC complex with PIM1 and its recruitment to MYC binding sites requires the MYC MBII domain (Fig. 2, 6), which is essential for MYC oncogenic activity, PIM1 was also fused to the transformationdefective MYC deleted in the MBII domain. Interestingly, we found that the fusion PIM1MYC MBII, but not co-transfection of MYC MBII with PIM1, was able to rescue, at least in part, the defective MYC-transformation (Fig. 8b). Importantly, this fusion rescues both transcriptional activity and ability to phosphorylate $\mathrm{H} 3 \mathrm{~S} 10$ at its recruiting sites (Fig. 8d, f). In contrast, a chimeric molecule in which PIM1 was fused to a MYC C, a mutant unable to bind to the DNA, was defective for transforming activity and for phosphorylation of $\mathrm{H} 3 \mathrm{~S} 10$ at the target genes analysed (see Supplementary Information Figure S8). Interestingly, the transformed foci induced by the PIM1-MYC fusion grew vigorously in soft agar forming bigger colonies as compared with PIM1KD-MYC (see Supplementary Information Fig. S8).

Finally, we tested whether $\mathrm{H} 3$ phosphorylation at S10 is required for MYC-dependent transformation by analysing MYC transforming potential in the presence of either yellow fluorescent protein (YFP) fused to $\mathrm{H} 3$ (YFP-H3) or to $\mathrm{H} 3$ mutated in S10 (YFP-H3S10A). Both fusion proteins co-localised in the nucleus during interphase and mitosis together with the endogenous H3S10ph (see Supplementary Information Fig. S9) and they did not interfere with cell cycle progression (see Supplementary Information Fig. S9) as previously reported for 
other histone fusion proteins ${ }^{37}$. Then we performed transformation assays by either expressing MYC, PIM1 or PIM1-MYC fusion proteins in these cells (see Supplementary Information Fig. S10). We observed that, while YFP-H3 did not affect the number of foci, YFP-H3S10A mutant significantly reduced the number of soft agar colonies, FOSL1 and ID2 transcription as well as $\mathrm{H} 3$ phosphorylation at their MYC binding sites (see Supplementary Information Fig. S10).

Taken together these results show that $\mathrm{H} 3$ phosphorylation at MYC-target genes by PIM1 is required for the cooperative effects of PIM1 and MYC in cell transformation.

\section{DISCUSSION}

This study defines a new role for the serine/threonine kinase PIM1 as a MYC-dependent modifier of the chromatin. Based on biochemical and functional evidence, we show that following growth factor stimulation a MYC/MAX/PIM1 complex, formed in the nucleus, recruits PIM1 to the chromatin where it phosphorylates H3 at S10. This PIM1-dependent nucleosome phosphorylation is required for the transcriptional activation of a subset of MYCtarget genes and MYC-dependent cell transformation.

Immunoprecipitation experiments showed that following growth factor stimulation MYC, in conjunction with MAX, forms a complex with PIM1 in the nucleus. Silencing experiments demonstrated that MYC is required for PIM1 recruitment to the chromatin while PIM1 is necessary to phosphorylate the $\mathrm{H} 3$ at $\mathrm{S} 10$ on the nuclesosme at $\mathrm{E}$ boxes to activate the transcription. According to the current view phosphorylation at the N-terminal domain of $\mathrm{H} 3$ could either be required to loosen the interaction between DNA and nucleosome and/or to 
generate a platform to recruit additional regulatory factors as described in the "histone code" hypothesis ${ }^{1}$. PIM1-dependent phosphorylation at the FOSL1 and ID2 genes is specific for its recruitment sites and does not spread over the genes, suggesting that this phosphorylation is necessary to trigger the enhancer to its active state.

It was previously shown that upon growth factors treatment $\mathrm{H} 3$ is phosphorylated at $\mathrm{S} 10$ with fast kinetics by MSK1/MSK2 ${ }^{4-7}$. Interestingly, FOSL1 shows two regions with different phosphorylation kinetics of H3S10: a phosphorylation site at the upstream SRE element, occurring within 15-30 minutes due to MSK1/MSK2, and a phosphorylation at the enhancer that was coincident with the peak of transcriptional activation of the gene mediated by PIM1. These two regions behaved differently with respect to acetylation of $\mathrm{H} 3$ at $\mathrm{K} 9$ and $\mathrm{K} 14$. While at the FOSL1 promoter $\mathrm{H} 3$ phosphorylation and acetylation are tightly coupled as previously shown for other IE promoters ${ }^{3,38-40}$, at the FOSL1 enhancer, phosphorylation of H3S10 takes place in a region highly acetylated at $\mathrm{H} 3$ prior to MYC and PIM1 binding in agreement with the finding that for MYC recognition its target sites must be highly acetylated in $\mathrm{H} 3$ as well as methylated in $\mathrm{H} 3 \mathrm{~K} 4 / \mathrm{K} 79^{41}$. On the FOSL1 enhancer phosphorylation and acetylation of $\mathrm{H} 3$ might have distinct functional roles as PIM1-dependent H3S10 phosphorylation is transient and limited to the E box element while acetylation at $\mathrm{K} 9$ and $\mathrm{K} 14$ is more stable and distributed along the gene suggesting that phosphorylation at these elements represents a critical step for the transcriptional activation. The fast turnover of $\mathrm{H} 3$ phosphorylation observed in our experiments and its temporal coincidence with transcriptional activation might be a general phenomenon as $\mathrm{H} 3$ phosphorylation was previously detected with the transcriptional activation of heat shock genes in Drosophila and with the RAR 2 gene induction by retinoic agonists in murine P19 embryonal cells while acetylation was 
constitutive at these $\operatorname{loci}^{42,43}$.

It has been estimated that about $11 \%$ of cellular genes present a functional $\mathrm{E}$ box to which MYC can associate on the genome ${ }^{28}$. MYC silencing demonstrated that MYC recruits PIM1 at specific MYC binding sites and confocal microscopy showed an elevated degree of nuclear colocalization of PIM1 with nascent transcripts and with MYC following growth factor treatment, suggesting that, at this stage of the cell cycle, PIM1 is recruited by MYC to a large number of sites. Gene expression profile analysis of MYC and PIM1 regulated genes in cells treated with serum at 120 minutes, revealed that 207 transcripts, corresponding to $20 \%$ of MYC regulated genes, also require PIM1 for their regulation. The co-regulation includes genes involved in cell metabolism, protein synthesis, cycle progression, and oncogenesis. Interestingly, a large number of genes are transcriptional factors, which suggests that PIM1 participates in MYCdependent regulatory networks. In agreement with these data cell cycle analysis, in PIM1 knockdown cells, revealed reduced entry in the $\mathrm{S}$ phase. Transformation analysis showed a more dramatic PIM1 effect as PIM1 silencing strongly inhibited the formation of MYCdependent transformed soft agar colonies. Although our experiments do not exclude that specific PIM1-regulated genes co-operate with MYC in cell transformation, our data strongly suggest that PIM1-dependent phosphorylation of H3 at MYC-target genes is necessary to regulate key genes required for MYC-dependent oncogenic transformation. In fact, PIM1 fusion to the transformation defective MYC MBII could significantly rescue its transforming potential as well as $\mathrm{H} 3$ phosphorylation at MYC binding sites while PIM1 co-expression with MYC MBII did not rescue.

In conclusion, this study provides a molecular mechanism for MYC and PIM1 cooperation in gene regulation and oncogenic transformation. As mutations that alter MYC expression are 
among the most common found in human and animal cancers ${ }^{44}$, it is conceivable that inhibiting MYC association with PIM1 and/or inhibiting PIM1 kinase activity by specific drugs might represent a method for the treatment of cancers in humans.

\section{METHODS}

DNA constructs. Human PIM1 and MYC cDNA were cloned into pcDNA5/FRT (Invitrogen) vector under the control of the CMV/TetO2 tetracycline inducible promoter. The PIM1-K67M construct was generated by introducing a site-specific mutation in the DNA sequence corresponding to the lysine 67 to mutate it into a methionine (K67M). The MYC MBII construct was obtained by deleting the DNA sequence corresponding to the amino acids 119152. The MYC C construct was obtained by deleting the DNA sequence corresponding to the amino acids 353-439. The PIM1-MYC fusion constructs were obtained by fusing the cDNA corresponding to PIM1 or PIM1-K67M via a 10 glycine encoding linker sequence to the cDNA corresponding either to MYC, MYC MBII or MYC C.

shRNA constructs were obtained by cloning a double-stranded DNA cassettes into the $\mathrm{pENTR/H1/TO}$ vector, which allows the expression of shRNA under the control of the tetracycline inducible $\mathrm{H} 1$ promoter/TetO2.

To generate shRNA cassettes the following oligonucleotides were designed following the manufacturer's protocol. The 21-nucleotides positive strands are:

PIM1 shRNA\#1 (shPIM1\#1): 5'-GTCTCTTCAGAGTGTCAGCAC-3'

PIM1 shRNA scrambled control\#1 (shsP\#1): 5'-GACTCATCAGTATGTTAGCAT-3'

PIM1 shRNA\#2 (shPIM1\#2): 5'-GAGGAAGAGAGTATCTATGGG 
PIM1 shRNA scramble control\#2 (shsP\#2): 5'-GAAGCCGAGAGTATCTATGTT -3' PIM1 shRNA\#3 (shPIM1\#3): 5'-CGAAGAAATCCAGGACCATCC -3' PIM1 shRNA scrambled control\#3 (shsP\#3): 5'-AAGTCATCCGAAGACCACAAC-3' PIM1 shRNA\#4 (shPIM1\#4): 5'-ACGTGGAGAAGGACCGGATTT-3' PIM1 shRNA scrambled control \#4 (shsP\#4): 5'-GATTCGAGGACATGGACTGAG-3' c-MYC shRNA\#1 (shMYC\#1): 5'-GCAATCACCTATGAACTTGTT-3' c-MYC shRNA scramble control\#1(shsM\#1): 5'-GCATTCACCTAAGAAGTTCTT-3' c-MYC shRNA\#2 (shMYC\#2): 5'- GACGACGAGACCTTCATCAAA -3' c-MYC shRNA2 scramble control \#2 (shMYC\#2): 5'-AGATCCAACCAGTCAGGACAT-3' c-MYC shRNA\#3 (shMYC\#3): 5'-CCTGAGACAGATCAGCAACAA-3' c-MYC shRNA scramble control\#3 (shsM\#3): 5'-CAACATGGTAACGAACAGCAA -3' c-MYC shRNA\#4 (shMYC\#4): 5'-CAGTTGAAACACAAACTTGAA-3' c-MYC shRNA scramble control\#4(shsM\#4): 5'-AACTAAGGTAACTATGCACAA-3' c-MYC shRNA\#5 (shMYC\#5): 5'-CCATAATGTAAACTGCCTCAA-3' c-MYC shRNA scramble control \#5(shsM\#5): 5'-GCAATGACCACCTATTCAATA-3' The recombinant GST-PIM1 construct was obtained by cloning the PIM1 WT and PIM1K67M cDNA into the bacterial expression vector pGEX-4T and the fused protein was expressed and purified using the standard protocol.

The vector coding for Xenopus leavis histones were expressed and purified as previously described ${ }^{45}$. Mutant recombinant histones H3 (H3S10A, H3S28A, H3S10A/S28A) were obtained by site directed mutagenesis as previously described ${ }^{46}$. YFP-H3 was kindly provided by Marco Bianchi and mutagenized as previously described ${ }^{46}$. 
Cell culture condition. We generated tetracycline inducible 293 cell line stably expressing PIM1 WT or PIM1-K67M by using the Invitrogen's Flp-T-Rex System.

pCDNA5/FRT/TO expression vector containing the gene of interest was inserted into the cells via Flp recombinase-mediated DNA recombination at the FRT site. The transfected cells were selected on the basis of hygromycin B (Sigma) resistance and pool clones were isolated.

Selected clones were cultivated in Dulbecco modified essential medium (D-MEM) containing $10 \%$ fetal bovine serum (FBS; Sigma) and $150 \mu \mathrm{g} / \mathrm{ml}$ hygromycin for $2 / 3$ weeks.

To perform time course experiments 293 cells were synchronized and then treated with $50 \%$ serum shock as described ${ }^{47}$. Primary HUVEC cells were grown in M199 medium supplemented with $15 \%$ fetal bovine serum, brain protein extracts and heparin $(50 \mathrm{~g} / \mathrm{ml})$. The cells were then starved for 24 hours in M199 medium containing 1\% FBS and 0.5\% BSA and induced with $50 \mathrm{ng} / \mathrm{ml}$ of VEGF-A.

The cell line P493-6 (kindly provided by Bruno Amati) were grown in RPMI 1640 containing $10 \%$ fetal calf serum (FCS) $100 \mathrm{U} / \mathrm{ml}$ penicillin, $100 \mu \mathrm{g} / \mathrm{ml}$ streptomycin $2 \mathrm{mM}$ L-glutammine. MYC repression was obtained with $0.1 \mu \mathrm{g} / \mathrm{ml}$ tetracycline. MYC induction was obtained by washing the cells three times with tetracycline fee phophate buffered saline containing $10 \%$ FCS as previously described ${ }^{27}$.

Immunostaining and co-localization analysis. Co-localization experiments were performed on fixed cells as previously described ${ }^{11}$ using a Leica TCS SP2 confocal microscopy. To label nascent RNA, anti-BrUTP (Sigma cat. B2531) has been utilized in permeabilized cells by immunodetection with small modifications from previously described protocol ${ }^{48}$. The degree of double staining was measured by quantitative analysis of the overlapping pixel using the 
Image $\mathbf{J} 1.32 \mathrm{j}$ (NIH, USA) software. All pixels having the same position in both images are considered coincident and the brightness level of each pixel is plotted as $\mathrm{x}$ and $\mathrm{y}$ axis of the scatter diagram. Two completely overlapping images will result in a straight diagonal line in scatter diagram.

Protein immunoprecipitation, interaction, kinase assays, chromatin immunoprecipitation. Proteins were extracted using F-buffer (10mM TRIS pH7.0, 50mMNaCl, 30mM Na $2 \mathrm{HPO}_{4}$, $50 \mathrm{mM} \mathrm{NaF}, 5 \mu \mathrm{M} \mathrm{ZnCl} 2,1 \%$ Triton-X 100) and treated with $1 \mathrm{U} / \mathrm{ml}$ Micrococcal nuclease (Sigma) at $4^{0} \mathrm{C}$ for $1 \mathrm{hr}$ to obtain the chromatin proteins (Chromatin fraction).

To identify interacting protein, $0.5 \mathrm{mg}$ of total proteins was incubated with $3 \mathrm{~g}$ of specific antibody overnight at $4^{\circ} \mathrm{C}$. Immunocomplexes were incubated with Protein A- or Gconjugated magnetic beads (DYNAL) for 30 minutes at $4^{\circ} \mathrm{C}$. Samples were washed four times with $\mathrm{F}$ buffer and 2 times with $0,15 \mathrm{M} \mathrm{NaCl} \mathrm{F-buffer} \mathrm{for} 20$ minutes at $4^{\circ} \mathrm{C}$. Proteins were eluded by boiling the samples and analysed by western blotting.

GST-interaction assay was performed by incubating 1mg of protein extracts (derived from chromatin fractions) with recombinant proteins GST, GST-PIM1WT and GST-PIM1-K67M bound to Glutathione-sepharose resin (Amersham). To allow specific interactions, proteins were incubated in Interaction buffer (TRIS 20mM; KCl 200mM; DTT 10mM; EGTA 0,1mM; Glycerol 20\%; Inhibitor protease cocktail) for 2 hours at $4{ }^{\circ} \mathrm{C}$ in presence of 21 -aa peptides. Unbound proteins were eliminated by 3 washes in the same buffer followed by protein equilibration in the Kinase buffer (TRIS 30mM; DTT 10mM; EGTA 0,1mM MnCl 2mM; Glycerol 10\%). Kinase assay was performed by incubating samples in the same buffer in presence of 4 pmol of $\left[\gamma^{32} \mathrm{P}\right]$ ATP for 2 minutes at $30^{\circ} \mathrm{C}$. Reaction was stopped by adding 
10mM EDTA and samples were washed 6 times with Kinase buffer containing 10mM EDTA and $200 \mathrm{mM} \mathrm{KCl}$. Phosphorylated proteins were separeted by SDS-PAGE and visualized by autoradiography.

Nucleosome core particles (NCPs) reconstitution was carry out as described ${ }^{49}$.

Chromatin Immunoprecipitation (ChIP) assays were performed as described by Upstate protocols (www.upstate.com). DNA was analyzed by quantitative real-time PCR by using Light Cycler DNA Amplification Kit SYBR Green I (Roche, Mannheim, Germany). All experiment values were normalized to those obtained with a non-immune serum and divided by input, using the procedure previously described ${ }^{50}$. Experiments of MSK1/MSK2 inhibition were performed by incubating cells with $\mathrm{H} 89$ (Calbiochem) as previously described ${ }^{5}$. Oligonucleotide sequences will be provided upon request.

Quantitative RT-PCR analysis was performed as previously described ${ }^{11}$.

\section{Cell transformation and colony formation in soft agarose}

The analysis of cell transformation and anchorage independent growth were performed as previously described ${ }^{36}$. Cells transfected with the constructs indicated were suspended at approximately $1 \times 10^{4}$ cells in $2 \mathrm{ml}$ of $0.35 \%$ low-melting agarose onto $35-\mathrm{mm}$ culture dish containing $0.7 \%$ agarose base. Triplicates were prepared for each tested construct and inspected by eye after crystal violet staining for colony formation after incubation at $37^{\circ} \mathrm{C}$ for 10-14 days. Efficiency of transfection was measured by reporter GFP gene and the levels of proteins were compared by Western blotting.

\section{Antibodies}


The antibodies used for in this work are purchased by Santa Cruz Biotechnology (anti-PIM1 SC-7856 for ChIP and immunofluorescence experiments; anti-PIM1 SC-13513 for Western blot analysis; anti-Myc SC-764 for ChIP and immunofluorescence experiments and anti-Myc SC-40 for Western blot analysis; anti-MAX SC-197 and SC-765; anti-SP1 SC-59; anti-Pol II SC-899), by Covance anti Pol II Ser2 H5, by Upstate (anti-Histone H3 06-755; anti-acetyl Histone H3 06-599; anti-phospho (Ser10)-acetyl (Lys14)-Histone H3 07-081; anti-phospho (Ser10)- Histone H3 05-817), and by Abcam anti phospho (Ser10)-acetyl (Lys9)-Histone H3 Ab 12181-50.

\section{ACKNOWLEDGEMENTS}

This work was supported by Associazione Italiana Ricerca sul cancro (AIRC), Ministero Italiano Universita' e Ricerca (MIUR), and Fondazione Monte dei Paschi di Siena. We are in indebted to all members of the laboratory for reagents, helpful suggestions, and encouragement; Marina Rocchigiani for VEGF-A preparations and Beatrice Grandi for technical support. Marco Bianchi for YFP-H3 construct, Roberto Mantovani for Xenopus leavis histone constructs, Bruno Amati for Rat-1 and P493-6 cells. Isabel Delany, Marco Bianchi, and Ernesto Guccione for the critical reading of the manuscript.

\section{AUTHOR CONTRIBUTION}

A.Z. planned and performed the experiments and analysed the data. A.D. generated stable clones and perfomed immunoprecipitation experiments. R.S. performed the transformation assays. S.O. planned the experimental design, analysed the data and wrote the manuscript.

\section{COMPETING FINANCIAL INTEREST}

The authors declare that they have no competing financial interests. 


\section{REFERENCES}

1. Strahl, B.D. \& Allis, C.D. The language of covalent histone modifications. Nature 403, 41-5 (2000).

2. Berger, S.L. Histone modifications in transcriptional regulation. Curr Opin Genet Dev 12, 142-8 (2002).

3. Cheung, P., Allis, C.D. \& Sassone-Corsi, P. Signaling to chromatin through histone modifications. Cell 103, 263-71 (2000).

4. Nowak, S.J. \& Corces, V.G. Phosphorylation of histone H3: a balancing act between chromosome condensation and transcriptional activation. Trends Genet 20, 214-20 (2004).

5. Thomson, S., Mahadevan, L.C. \& Clayton, A.L. MAP kinase-mediated signalling to nucleosomes and immediate-early gene induction. Semin Cell Dev Biol 10, $205-$ 14 (1999).

6. Sassone-Corsi, P. et al. Requirement of Rsk-2 for epidermal growth factor-activated phosphorylation of histone H3. Science 285, 886-91 (1999).

7. Soloaga, A. et al. MSK2 and MSK1 mediate the mitogen- and stress-induced phosphorylation of histone H3 and HMG-14. Embo J 22, 2788-97 (2003).

8. Yamamoto, Y., Verma, U.N., Prajapati, S., Kwak, Y.T. \& Gaynor, R.B. Histone H3 phosphorylation by IKK-alpha is critical for cytokine-induced gene expression. Nature 423, 655-9 (2003). 
9. Anest, V. et al. A nucleosomal function for IkappaB kinase-alpha in NF-kappaBdependent gene expression. Nature 423, 659-63 (2003).

10. Cuypers, H.T. et al. Murine leukemia virus-induced T-cell lymphomagenesis: integration of proviruses in a distinct chromosomal region. Cell 37, 141-50 (1984).

11. Zippo, A., De Robertis, A., Bardelli, M., Galvagni, F. \& Oliviero, S. Identification of Flk-1-target genes in vasculogenesis: Pim-1 is required for endothelial and mural cell differentiation in vitro. Blood 103, 4536-44 (2004).

12. van Lohuizen, M. et al. Predisposition to lymphomagenesis in pim-1 transgenic mice: cooperation with c-myc and N-myc in murine leukemia virus-induced tumors. Cell 56, 673-82 (1989).

13. van Lohuizen, M. et al. Identification of cooperating oncogenes in E mu-myc transgenic mice by provirus tagging. Cell 65, 737-52 (1991).

14. Verbeek, S. et al. Mice bearing the E mu-myc and E mu-pim-1 transgenes develop pre-B-cell leukemia prenatally. Mol Cell Biol 11, 1176-9 (1991).

15. Wang, Z. et al. Pim-1: a serine/threonine kinase with a role in cell survival, proliferation, differentiation and tumorigenesis. J Vet Sci 2, 167-79 (2001).

16. Ellwood-Yen, K. et al. Myc-driven murine prostate cancer shares molecular features with human prostate tumors. Cancer Cell 4, 223-38 (2003).

17. Amati, B., Alevizopoulos, K. \& Vlach, J. Myc and the cell cycle. Front Biosci 3, d250-68 (1998).

18. Grandori, C., Cowley, S.M., James, L.P. \& Eisenman, R.N. The Myc/Max/Mad network and the transcriptional control of cell behavior. Annu Rev Cell Dev Biol 16, 653-99 (2000). 
19. Levens, D.L. Reconstructing MYC. Genes Dev 17, 1071-7 (2003).

20. Murphy, M.J., Wilson, A. \& Trumpp, A. More than just proliferation: Myc function in stem cells. Trends Cell Biol 15, 128-37 (2005).

21. Grandori, C. et al. c-Myc binds to human ribosomal DNA and stimulates transcription of rRNA genes by RNA polymerase I. Nat Cell Biol 7, 311-8 (2005).

22. Arabi, A. et al. c-Myc associates with ribosomal DNA and activates RNA polymerase I transcription. Nat Cell Biol 7, 303-10 (2005).

23. Trumpp, A. et al. c-Myc regulates mammalian body size by controlling cell number but not cell size. Nature 414, 768-73 (2001).

24. Adhikary, S. \& Eilers, M. Transcriptional regulation and transformation by Myc proteins. Nat Rev Mol Cell Biol 6, 635-45 (2005).

25. McMahon, S.B., Van Buskirk, H.A., Dugan, K.A., Copeland, T.D. \& Cole, M.D. The novel ATM-related protein TRRAP is an essential cofactor for the c-Myc and E2F oncoproteins. Cell 94, 363-74 (1998).

26. Osborne, C.S. et al. Active genes dynamically colocalize to shared sites of ongoing transcription. Nat Genet 36, 1065-71 (2004).

27. Schuhmacher, M. et al. The transcriptional program of a human B cell line in response to Myc. Nucleic Acids Res 29, 397-406 (2001).

28. Fernandez, P.C. et al. Genomic targets of the human c-Myc protein. Genes Dev 17, $1115-29(2003)$.

29. Bergers, G., Graninger, P., Braselmann, S., Wrighton, C. \& Busslinger, M. Transcriptional Activation of the Fra-1 gene by AP-1 is mediated by regulatory sequences in the first intron. Mol. Cell. Biol. 15, 3748-3758 (1995). 
30. Adiseshaiah, P., Peddakama, S., Zhang, Q., Kalvakolanu, D.V. \& Reddy, S.P. Mitogen regulated induction of FRA-1 proto-oncogene is controlled by the transcription factors binding to both serum and TPA response elements. Oncogene 24, 4193-205 (2005).

31. Blackwell, T.K. et al. Binding of myc proteins to canonical and noncanonical DNA sequences. Mol Cell Biol 13, 5216-24 (1993).

32. Lasorella, A., Noseda, M., Beyna, M., Yokota, Y.\& Iavarone, A. Id2 is a retinoblastoma protein target and mediates signalling by Myc oncoproteins. Nature 407, 592-8 (2000).

33. Zeller, K.I. et al. Global mapping of c-Myc binding sites and target gene networks in human B cells. Proc Natl Acad Sci U S A 103, 17834-9 (2006).

34. Schuhmacher, M. et al. Control of cell growth by c-Myc in the absence of cell division. Curr Biol 9, 1255-8 (1999).

35. Pajic, A. et al. Cell cycle activation by c-myc in a burkitt lymphoma model cell line. Int J Cancer 87, 787-93 (2000).

36. Eilers, M., Picard, D., Yamamoto, K.R. \& Bishop, J.M. Chimaeras of myc oncoprotein and steroid receptors cause hormone-dependent transformation of cells. Nature 340, 66-8 (1989).

37. Kanno, T. et al. Selective recognition of acetylated histones by bromodomain proteins visualized in living cells. Mol Cell 13, 33-43 (2004).

38. Barratt, M.J., Hazzalin, C.A., Cano, E. \& Mahadevan, L.C. Mitogen-stimulated phosphorylation of histone $\mathrm{H} 3$ is targeted to a small hyperacetylation-sensitive fraction. Proc Natl Acad Sci U S A 91, 4781-5 (1994). 
39. Cheung, P. et al. Synergistic coupling of histone H3 phosphorylation and acetylation in response to epidermal growth factor stimulation. Mol Cell 5, 905-15 (2000).

40. Clayton, A.L., Rose, S., Barratt, M.J. \& Mahadevan, L.C. Phosphoacetylation of histone $\mathrm{H} 3$ on c-fos- and c-jun-associated nucleosomes upon gene activation. Embo $J$ 19, 3714-26 (2000).

41. Guccione, E. et al. Myc-binding-site recognition in the human genome is determined by chromatin context. Nat Cell Biol (2006).

42. Nowak, S.J. \& Corces, V.G. Phosphorylation of histone H3 correlates with transcriptionally active loci. Genes Dev 14, 3003-13 (2000).

43. Lefebvre, B., Ozato, K. \& Lefebvre, P. Phosphorylation of histone H3 is functionally linked to retinoic acid receptor beta promoter activation. EMBO Rep 3, $335-40$ (2002).

44. Cole, M.D. \& McMahon, S.B. The Myc oncoprotein: a critical evaluation of transactivation and target gene regulation. Oncogene 18, 2916-24 (1999).

45. Caretti, G., Motta, M.C. \& Mantovani, R. NF-Y associates with H3-H4 tetramers and octamers by multiple mechanisms. Mol Cell Biol 19, 8591-603 (1999).

46. Salameh, A., Galvagni, F., Bardelli, M., Bussolino, F. \& Oliviero, S. Direct recruitment of CRK and GRB2 to VEGFR-3 induces proliferation, migration, and survival of endothelial cells through the activation of ERK, AKT, and JNK pathways. Blood 106, 3423-31 (2005).

47. Balsalobre, A., Damiola, F. \& Schibler, U. A serum shock induces circadian gene expression in mammalian tissue culture cells. Cell 93, 929-37 (1998). 
48. Zhang, S., Herrmann, C. \& Grosse, F. Nucleolar localization of murine nuclear DNA helicase II (RNA helicase A). J Cell Sci 112 ( Pt 16), 2693-703 (1999).

49. Cirillo, L.A. \& Zaret, K.S. Preparation of defined mononucleosomes, dinucleosomes, and nucleosome arrays in vitro and analysis of transcription factor binding. Methods Enzymol 375, 131-58 (2004).

50. Kouskouti, A. \& Talianidis, I. Histone modifications defining active genes persist after transcriptional and mitotic inactivation. Embo J 24, 347-57 (2005).

\section{FIGURE LEGENDS}

Figure 1. PIM1 phosphorylates H3S10 on the nucleosome.

(a) PIM1 phosphorylates histone $\mathrm{H} 3$ on nucleosomes from chromatin fraction. GSTrecombinant proteins, as indicated, were incubated with purified nucleosomes from 293 cells and the kinase reactions were carried out in the presence of $\left[\gamma^{32} P\right]$ ATP. GST-PIM1 interactions with nucleosomes were performed in the absence (-) or presence of 20 fold molar excess of unrelated (unr), H3 or H2B peptides as indicated. GST recombinant proteins and nucleosomes were visualized by Coomassie staining. Histone phosphorylation was revealed by autoradiography. $\mathrm{H} 3$ but not $\mathrm{H} 2 \mathrm{~B}$ N-terminal domain competes for PIM1 association to the nucleosome inhibiting the kinase reaction.

(b) PIM1 phosphorylates histone $\mathrm{H} 3$ at serine 10 . Recombinant GST-PIM1 or the PIM1 kinase inactive mutant were incubated with nucleosomes reconstituted in vitro from recombinant histones. Nucleosomes were assembled containing respectively the 
histone H3 wild type (lanes 1, 2), the mutant H3S10A (lanes 3,4), the mutant H3S28A (lanes 5,6) or the double mutant H3S10A/S28A (lanes 7,8). Histone H3 autoradiography resulting from $\left[\gamma_{-}{ }^{32} \mathrm{P}\right]$ ATP incorporation (first panel) is shown. The input kinases and nuclesomes were visualized by Coomassies staining (lower panels). (c) PIM1 interacts with histone H3 in vivo. PIM1 was immunoprecipitated from 293 stable clones expressing the inducible PIM1 (WT) or the kinase inactive mutant PIM1K67M (K67M) as indicated. PIM1 and H3 were revealed by immunoblotting (IB) with specific antibodies. A kinase reaction (KA) was carried out incubating the PIM1/H3 coimmunoprecipitate in the presence of $\left[\tilde{\gamma}^{32} \mathrm{P}\right]$ ATP. Histone phosphorylation was visualized by autoradiography. $2 \%$ of the total immunoprecipitated protein samples were loaded as inputs (lower panel).

Figure 2. PIM1 forms a complex with MYC.

(a) PIM1 co-localizes with MYC in HUVEC upon VEGF-A treatment. Indirect immunofluorescence analysis of HUVEC treated with VEGF was performed by using antibodies against PIM1 (red) and MYC (green) and double immunostaining are shown $($ Scale bar $=70 \mu \mathrm{m})$.

(b) VEGF-A treatment induced interaction of endogenous PIM1 with the MYC/MAX dimer. Cell proteins extracted from VEGF-A treated HUVEC were either immunostained (INPUT) or subjected to immunoprecipitation (IP) with anti-lgG antibodies (-), or anti-MAX antibodies as indicated (+). Immunostaining (IB) analysis was performed by using anti-MAX, -MYC and -PIM1 antibodies as indicated. 10\% of the total immunoprecipitated protein samples were loaded as inputs. 
(c) Endogenous PIM1 forms a complex with MYC/MAX in serum-stimulated 293. Western Blot analysis of input extracts, IgG, MAX, MYC or PIM1 immunoprecipitates from serum induced or not induced as indicated. Interacting proteins were revealed by using anti-MAX, -MYC, or -PIM1 antibodies respectively.

(d) MYC forms a complex with PIM1 through its MBII domain. 293 were transiently cotransfected with FLAG-MYC constructs as indicated and PIM1. Cell lysates were immunoprecipitated with anti-IgG, anti-FLAG, or anti-PIM1 antibodies followed by Western blot analysis to detect MAX, MYC, PIM1 and TRRAP proteins as indicated. Asterisk indicated an unspecific band.

Figure 3. PIM1 co-localizes with H3S10ph, active chromatin, and MYC.

(a) Immunofluorescence analysis of PIM1 nuclear sub-localization in HUVEC treated with VEGF for 60 minutes. Fixed cells were double-stained for PIM1 (red) and one of the indicated markers (green) (Scale bar $=8 \mu \mathrm{m})$. The corresponding pictures were merged and all pixels having the same position in both images are considered coincident and were represented as white spots (co-localization points). The brightness level of each pixel is plotted as $x$ (PIM1) and y (marker) axis of the scatter diagram of the correlation plot. The pixels co-localization results of 7 individual images represented in the correlation plot were quantified as Pearson's correlation coefficient $(\mathrm{Rr})$ as mean $\pm \mathrm{SD}$ with $\mathrm{P}$ values determined using Student's $t$-test.

(b) Immunofluorescence analysis of MYC localization at transcriptional active sites. Cells were labeled with BrUTP and detected with anti-bromodeoxyuridine antibody 
(green). Double labeling was revealed at transcriptional active sites by staining cells with an anti-MYC antibody (red). The pixels co-localization were performed as in (a).

Figure 4. PIM1 associates to chromatin at the FOSL1 enhancer.

(a) FOSL1 is transiently induced by VEGF-A treatment. Serum-starved HUVEC were treated with VEGF-A and total RNAs were collected at different time points. FOSL1 transcript levels were quantified by quantitative RT-PCR and relative values normalized on GAPDH expression level. A representative experiment is shown. The data represent triplicate real-time quantitative RT-PCR measurements.

(b) Schematic representation of the FOSL1 gene region including the first two exons (not in scale). Exons are represented as black squares within the gene and the $E$ box is figured as a black spot. The probes used in ChIP analysis are indicated. PCR probes intervals are numbered relatively to the fist nucleotide of exon $1(+1)$.

(c) VEGF-A induces PIM1 association with chromatin at the FOSL1 enhancer. Protein interactions on FOSL1 chromatin were measured by quantitative ChIP assay on VEGF-A-treated HUVEC. Chromatin extracts were immunoprecipitated using antibodies as indicated. A representative experiment is shown. The data represent triplicate real-time quantitative PCR measurements of the immunoprecipitated DNA of the corresponding gene regions identified as probes $A, B, C$ or D at different time points and expressed as 1/1000 (\%) of the DNA inputs.

(d) FOSL1 expression in the presence of MSK1/MSK2 inhibitor H89. Serum-starved HUVEC were treated with VEGF-A in the presence or absence of $10 \mu \mathrm{M} \mathrm{H} 89$ and total RNAs were collected at different time points. FOSL1 transcript levels were quantified 
by real-time quantitative RT-PCR and relative values normalized on GAPDH expression level. A representative experiment is shown. The data represent triplicate real-time quantitative RT-PCR measurements.

(e) Phosphorylation of $\mathrm{H} 3 \mathrm{~S} 10$ at the FOSL1 upstream element is dependent from MSK1/MSK2 kinase activities. H3S10 phosphorylation was measured by quantitative ChIP assay on VEGF-A-treated HUVEC. Chromatin extracts were immunoprecipitated using antibodies as indicated. A representative experiment is shown. The data represent triplicate real-time quantitative PCR measurements of the immunoprecipitated DNA of the corresponding gene regions identified as probes $A, B$, C or D at different time points and expressed as 1/1000 (\%) of the DNA inputs.

Figure 5. PIM1 mediates $\mathrm{H} 3 \mathrm{~S} 10$ phosphorylation and contributes to FOSL1 transcription.

(a) Inducible shRNA expression targeting PIM1. Western blot analysis of 293 stable clones expressing a control scrambled shRNA (shsP\#1) or PIM1 shRNA (shPIM1\#1) inducible by tetracycline. Immunostaining with antibodies as indicated. Protein extracts were obtained from not treated or serum-treated cells as indicated, in presence $($ Tet +$)$ or absence (Tet -).

(b) PIM1 knockdown alters H3S10 phosphorylation at the FOSL1 enhancer. Probes A, $\mathrm{B}, \mathrm{C}$ and $\mathrm{D}$ are schematized as in Figure 4 . Time course analysis by ChIP assay was performed by using antibodies as specified from 293 cells expressing either a control scrambled shRNA or PIM1 shRNA. A representative experiment is shown. The data 
represent triplicate real-time quantitative PCR measurements of the immunoprecipitated DNA and expressed as 1/1000 (\%) of the DNA inputs.

Figure 6. PIM1 phosphorylates histone H3 in a MYC-dependent manner.

(a) Inducible MYC gene silencing. 293 were transiently transfected with either a control scrambled shRNA (shsM\#1) or MYC shRNA (shMYC\#1) as indicated by tetracycline treatment. Western blot analysis on protein extracts was performed by immunoblotting, using antibodies recognising MYC, MAX, or SP1 as indicated.

(b-e) MYC recruits PIM1 on chromatin. Chromatin samples were obtained from serum-induced 293 cells expressing the control scrambled shRNA or MYC shRNA as indicated. ChIP assays were performed using antibodies as indicated. Immunoprecipitated DNA was analyzed by quantitative PCR with primers to amplify the FOSL1 enhancer and ID2 upstream MYC-bindig sites (-1705/-1460). A representative experiment is shown. The data represent triplicate real-time quantitative PCR measurements of the immunoprecipitated DNA and expressed as $1 / 1000(\%)$ of the DNA inputs.

(f) Western blot analysis of 293 transiently transfected with control scrambled shRNA (shsM\#1), MYC shRNA (shMYC\#1), or the shRNA together with plasmids expressing either FLAG-MYC or FLAG-MYC MBII as indicated. shMYC\#1 recognizes MYC 3'untranslated region which is not present in the FLAG constructs co-transfected. Protein expression was performed by immunoblotting, using antibodies as indicated. (g-h) MYC but not FLAG-MYC BMII rescues the recruitment of PIM1 to the chromatin. Chromatin samples were obtained from 293 cells expressing the control 
scrambled shRNA, MYC shRNA, or the shRNA together with FLAG-MYC or FLAGMYC MBII (F-MYC MBII) as indicated. ChIP assays were performed using antibodies as indicated. Immunoprecipitated DNA was analyzed by quantitative PCR with primers to amplify the FOSL1 enhancer and ID2 upstream MYC-binding sites (1705/-1460). A representative experiment is shown. The data represent triplicate realtime quantitative PCR measurements of the immunoprecipitated DNA and expressed as $1 / 1000(\%)$ of the DNA inputs.

Figure 7. PIM1 is required for the transcriptional activation of FOSL1 and ID2.

(a) Schematic representation of the FOSL1 gene region including the first two exons (not in scale). Exons are represented as black squares within the gene and the $E$ box is figured as a black spot. The probes used in ChIP analysis are indicated. PCR probes intervals are numbered relatively to the fist nucleotide of exon $1(+1)$.

(b) Time-course analysis by quantitative real time RT-PCR of FOSL1 mRNA and unprocessed heterogeneous nuclear FOSL1 RNA (hnRNA) in 293 cells expressing either a control scrambled shRNA or PIM1 shRNA as indicated. Gene specific RNA levels at each time point were measured and normalized on GAPDH expression and represented as fold induction (vertical bars). A representative experiment is shown. The data represent triplicate real-time quantitative RT-PCR measurements.

(c) Time course analysis by ChIP assay was performed by using antibodies recognising RNA Polimerase II (Pol II) or RNA Polimerase II phosphorylated in its C terminal domain at Serine 2 (Ser2P) from 293 cells expressing either a control scrambled shRNA or PIM1 shRNA at the FOSL1 promoter or coding region as 
specified in panel A. A representative experiment is shown. The data represent triplicate real-time quantitative PCR measurements of the immunoprecipitated DNA and expressed as 1/1000 (\%) of the DNA inputs.

(d) Schematic representation of the ID2 gene region including the first two exons (not in scale). Exons are represented as black rectangles within the gene and the $E$ box is figured as a black spot. The probes used in ChIP analysis are indicated. PCR probes intervals are numbered relatively to the fist nucleotide of exon $1(+1)$.

(e) Time-course analysis by quantitative real time RT-PCR of ID2 mRNA and ID2 hnRNA in 293 cells expressing either a control scrambled shRNA (shsP\#1) or PIM1 shRNA (shPIM1 \#1). Gene specific RNA levels at each time point were measured and normalized on GAPDH expression and represented as fold induction (vertical bars). A representative experiment is shown. The data represent triplicate real-time quantitative RT-PCR measurements.

(f) Time course analysis by ChIP assay was performed by using antibodies recognising RNA Polimerase II (Pol II) or RNA Polimerase II phosphorylated in its C terminal domain at Serine 2 (Ser2P) from 293 cells expressing either a control scrambled shRNA or PIM1 shRNA at the ID2 promoter or coding region as specified in panel $D$.

A representative experiment is shown. The data indicated represent triplicate real-time quantitative PCR measurements of the immunoprecipitated DNA and expressed as $1 / 1000(\%)$ of the DNA inputs. 
Figure 8. PIM1 contributes to MYC-dependent transformation. (a b) Soft agar colony formation of Rat-1 cell lines. Rat-1 fibroblasts were transfected with the constructs indicated. Transforming potential of the transfected constructs was determined by examining the soft agar colonies formed within two weeks post-transfection. Transforming potential was estimated by the average number of visible soft agar colonies observed in three plates. A representative experiment is shown.

(c-d) Quantitative real time RT-PCR of FOSL1 and ID2 mRNA in Rat-1 cells expressing the constructs indicated. Gene specific RNA levels were measured and normalized on GAPDH expression and represented as fold induction (vertical bars). A representative experiment is shown. The data represent triplicate real-time quantitative RT-PCR measurements.

(e-f) ChIP assay performed in Rat-1 cells by using antibodies as indicated. The primers used for the FOSL1 gene correspond to probe B shown in Figure 4 and for the ID2 MYC binding sites as in Figure 7. A representative experiment is shown. The data represent triplicate real-time quantitative PCR measurements of the immunoprecipitated DNA and expressed as 1/1000 (\%) of the DNA inputs. 
a

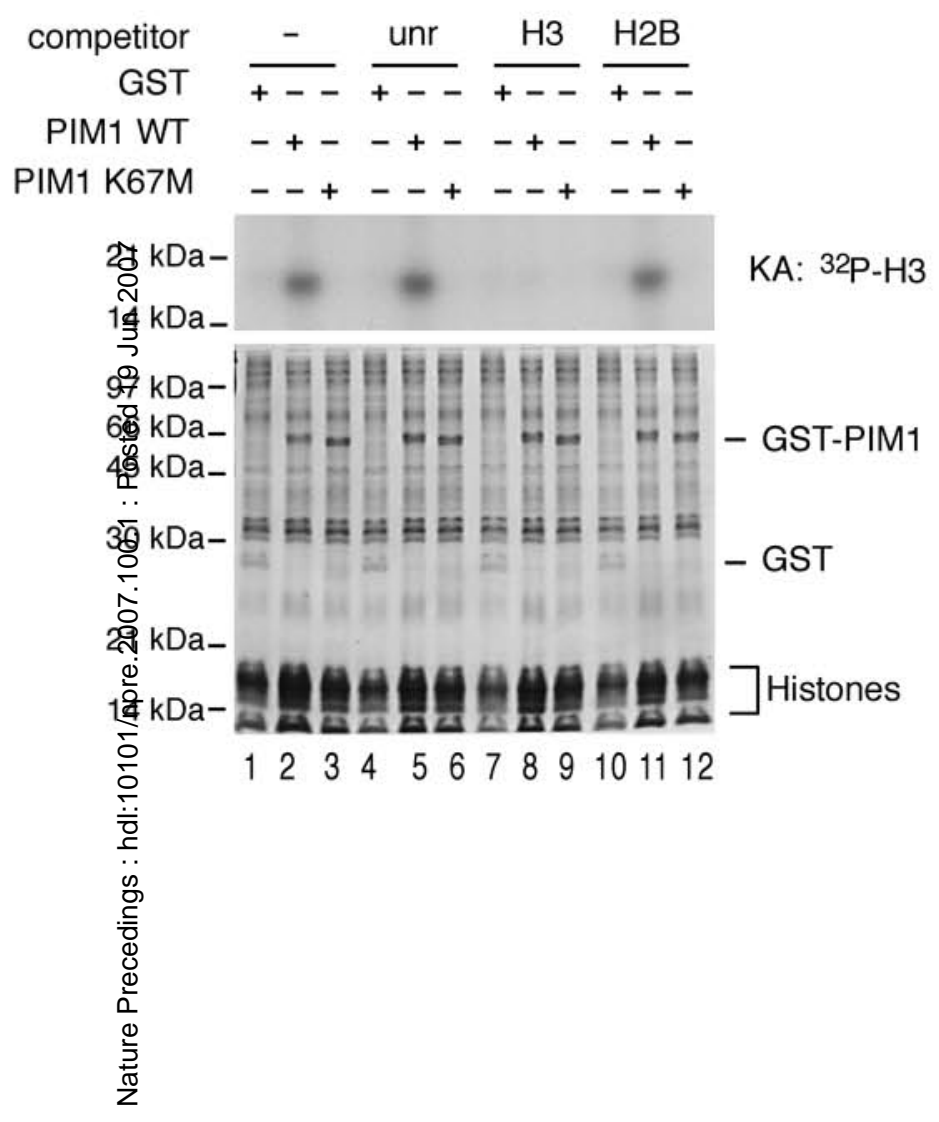

b

C

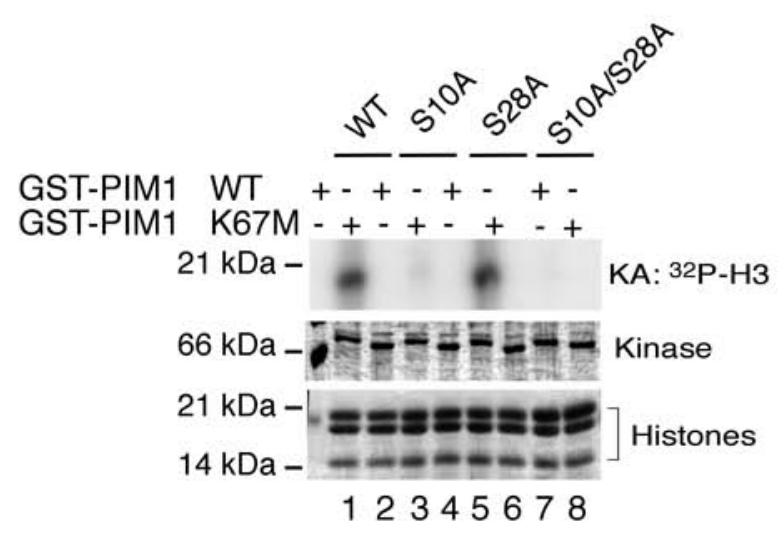

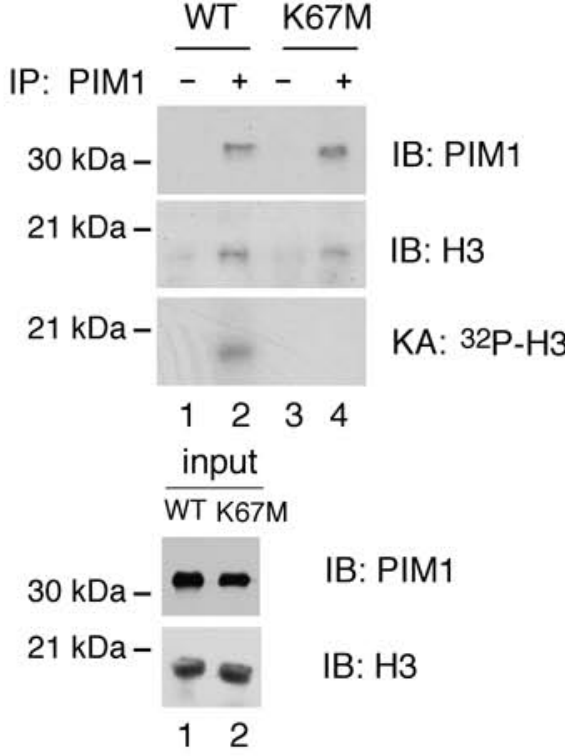

Fig. 1 

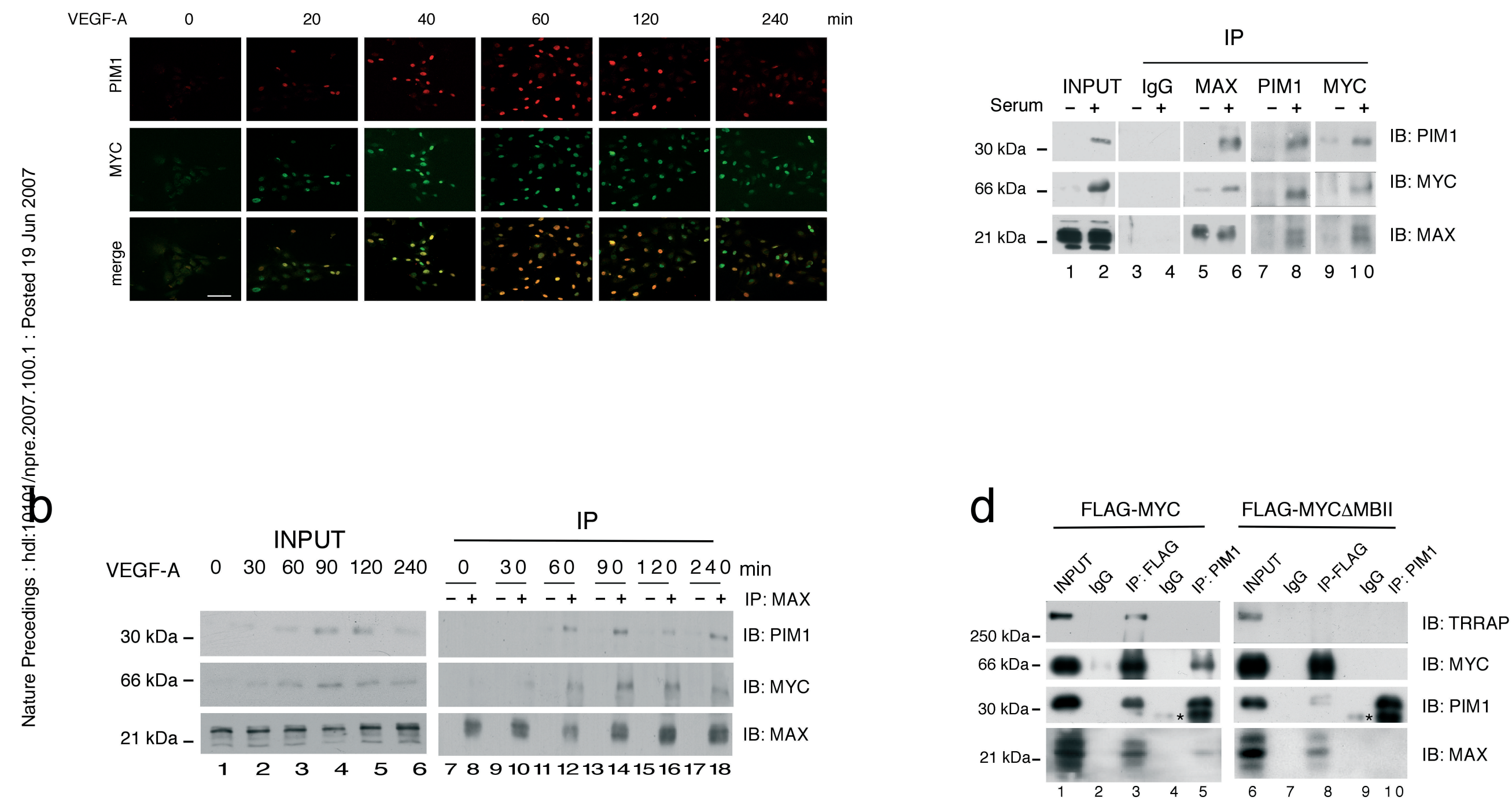

Fig. 2 
a

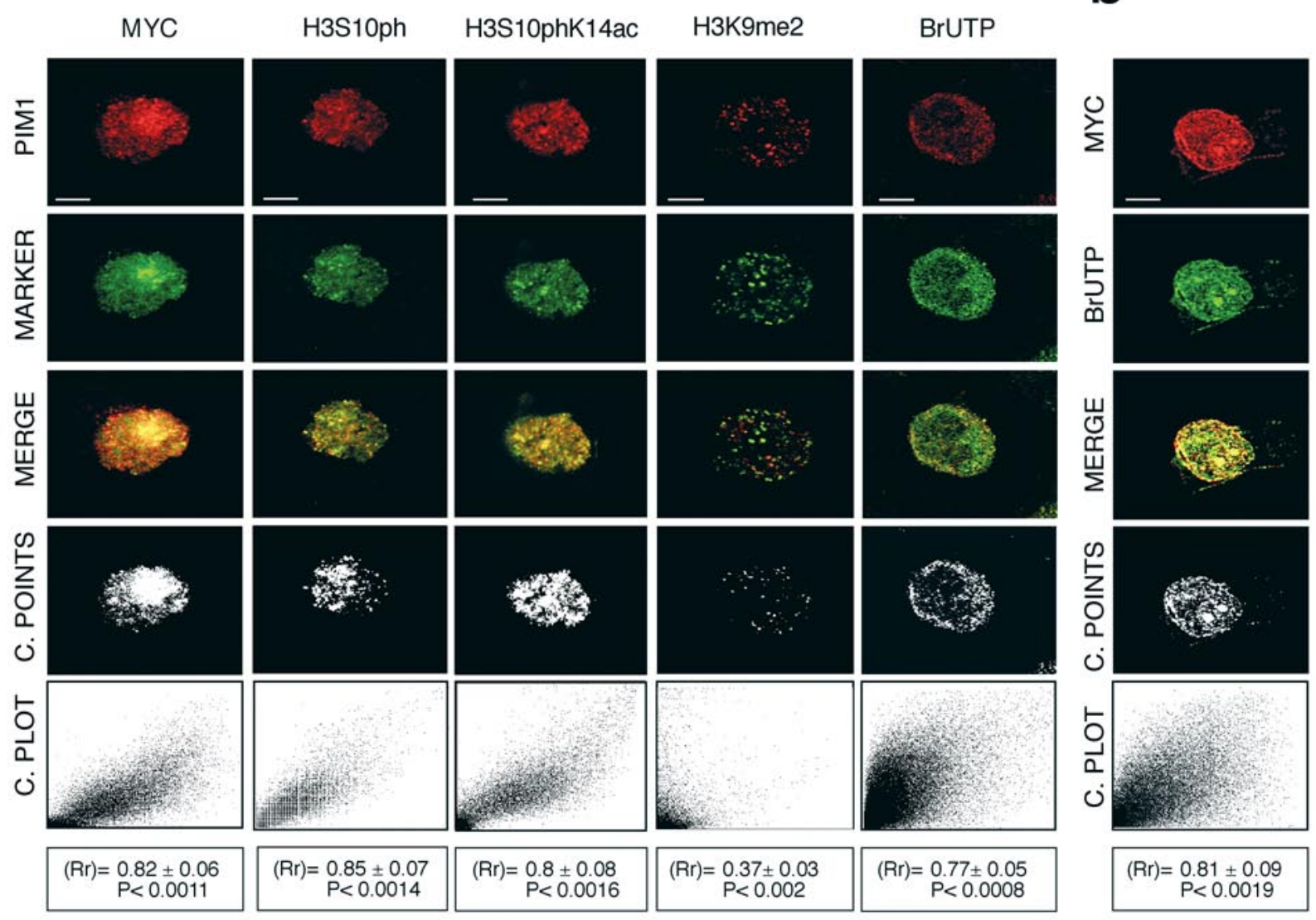

Fig.3 


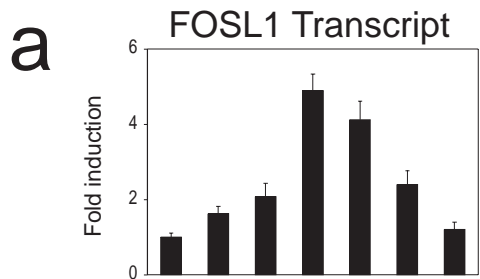

$\begin{array}{lllllllll}\text { VEGF-A } & 0 & 15 & 30 & 60 & 90 & 120 & 240 & \mathrm{~min}\end{array}$ b

Probe A

$(-590 /-330)$

FOSL1

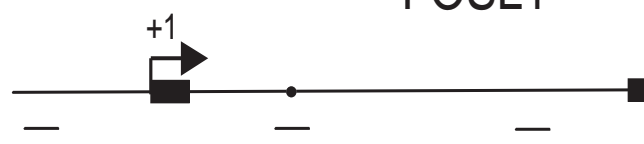

B

$(+930 /+1170)$
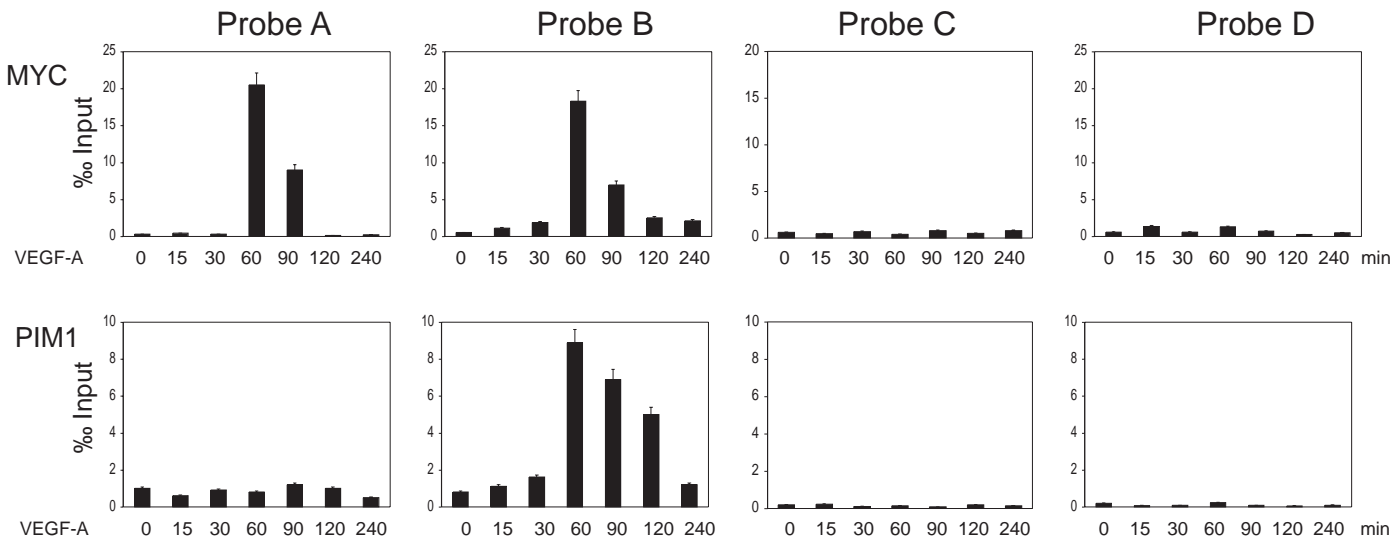

H3S10ph
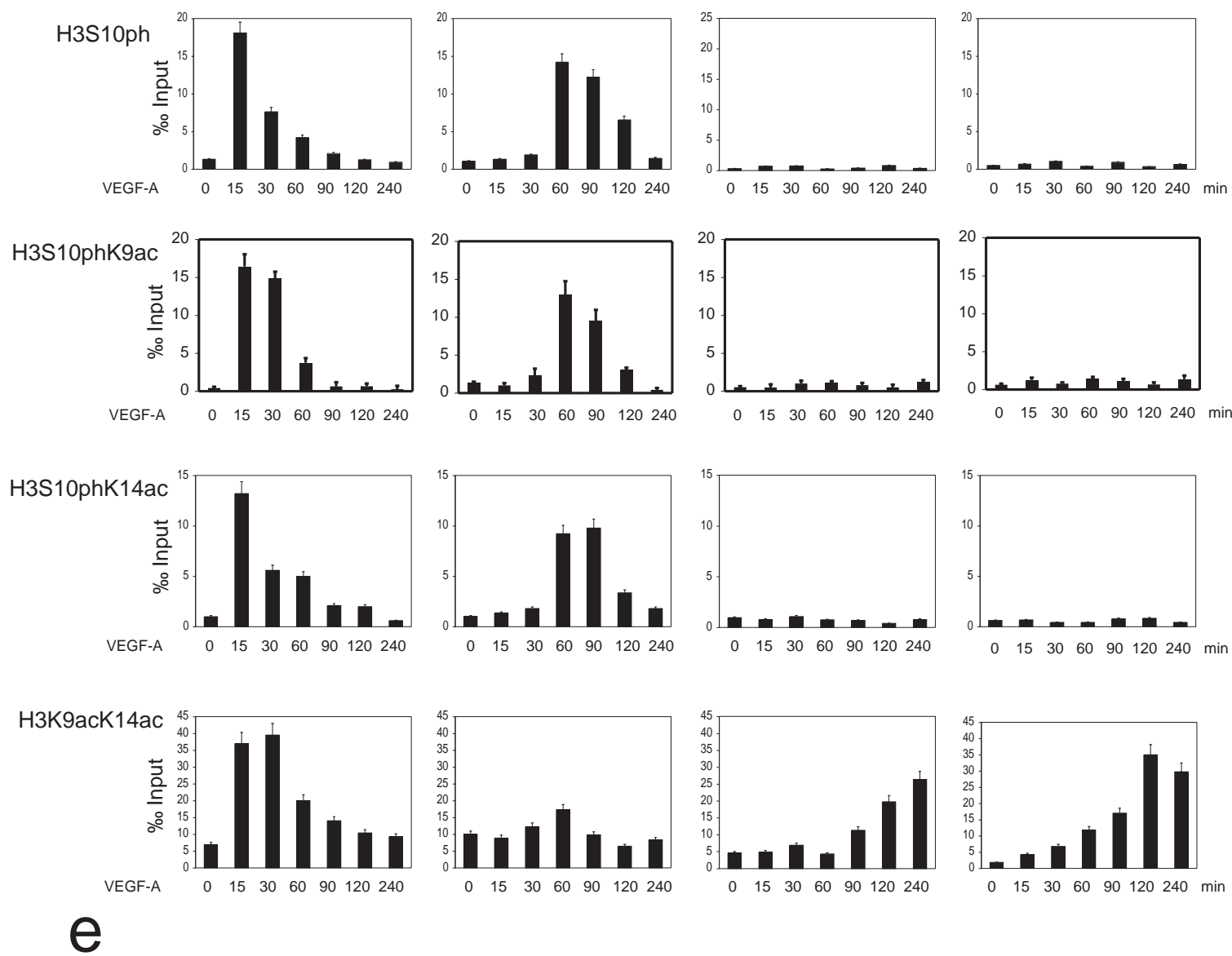

d
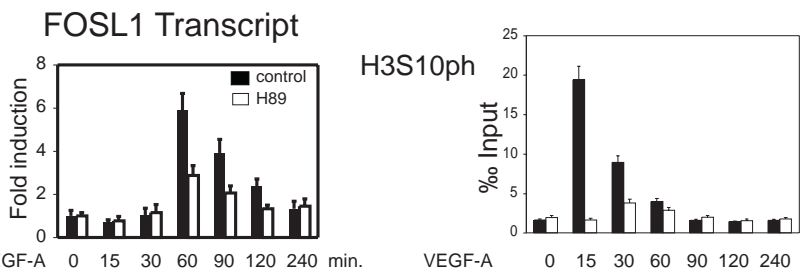
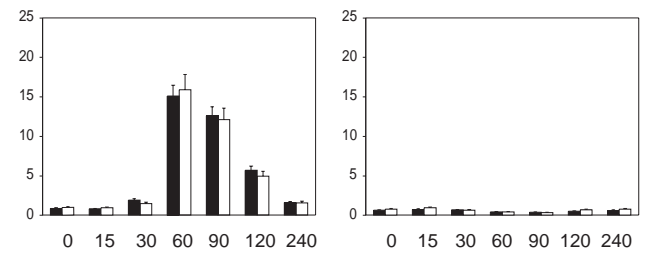


\section{a}

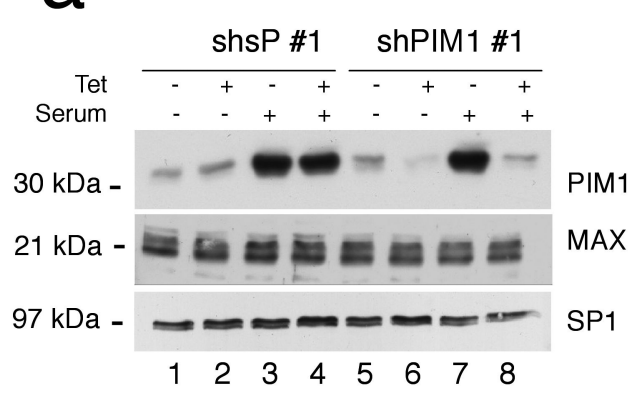

\section{b} H3S10ph

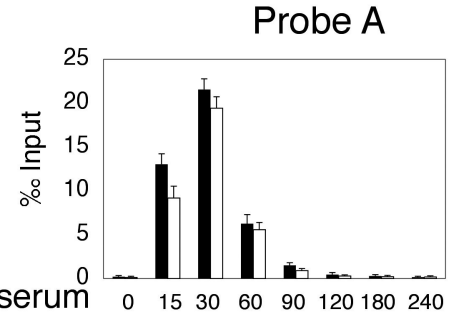

PIM1
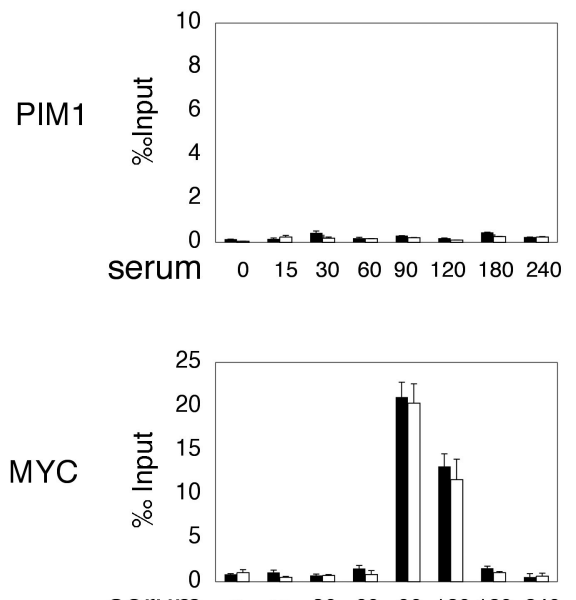

H3S10phK9ac

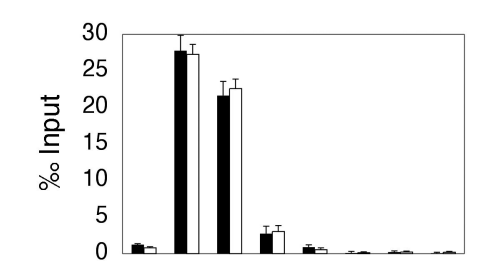

serum $\quad \begin{array}{lllllll}15 & 30 & 60 & 90 & 120180 & 240\end{array}$

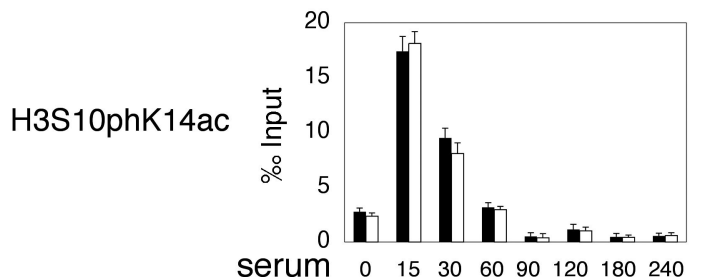

Fig. 5

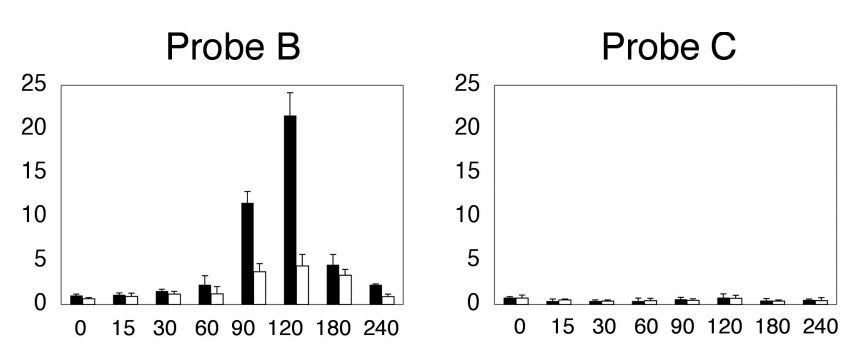

Probe D
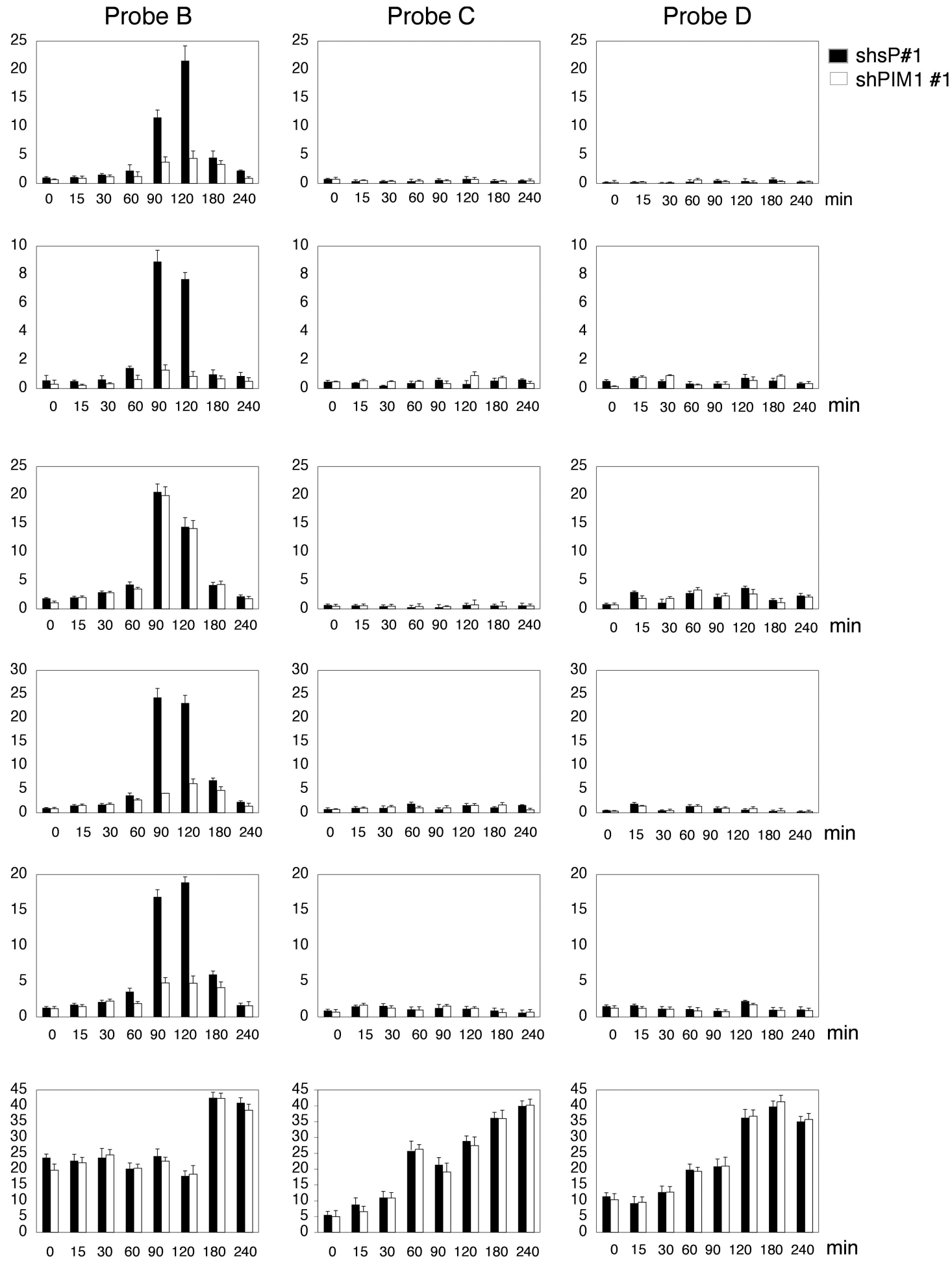
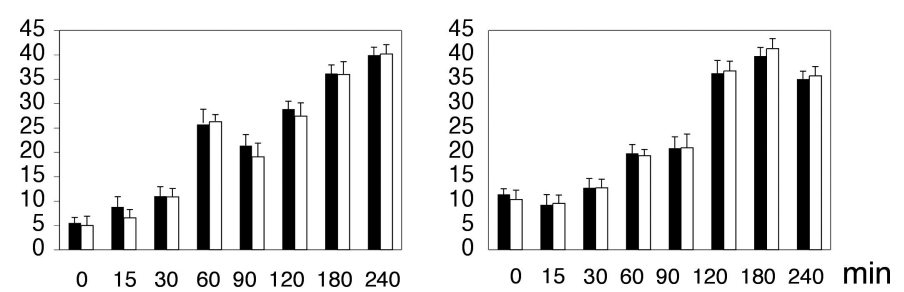
a

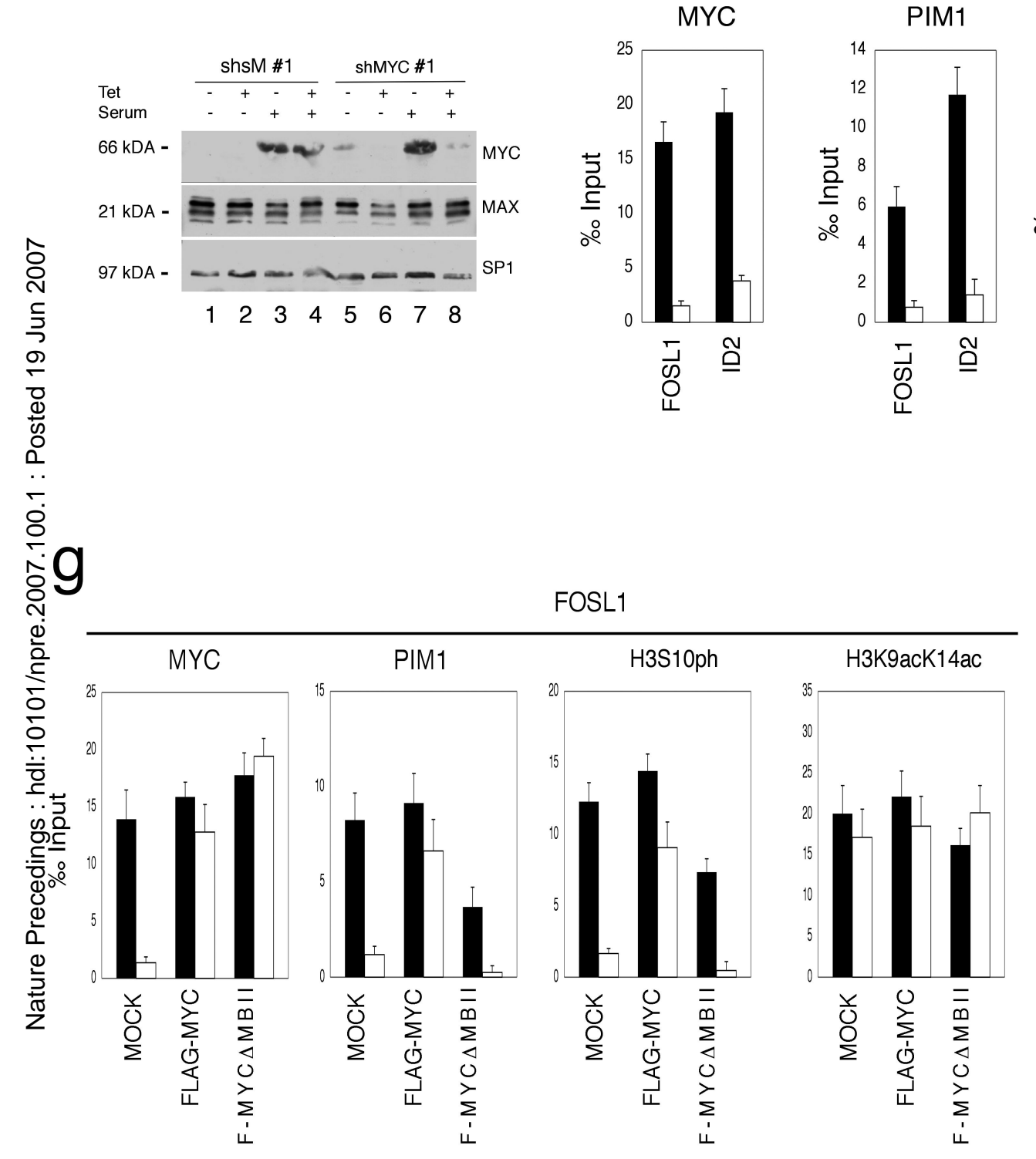

d e

H3S10ph
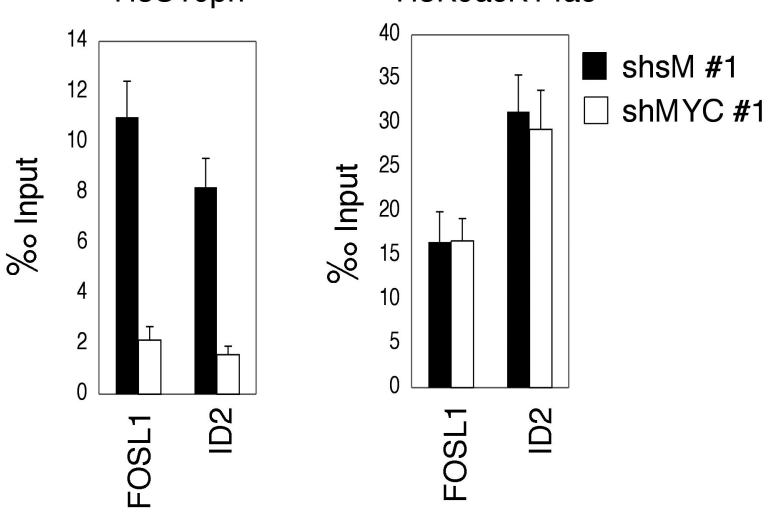

h

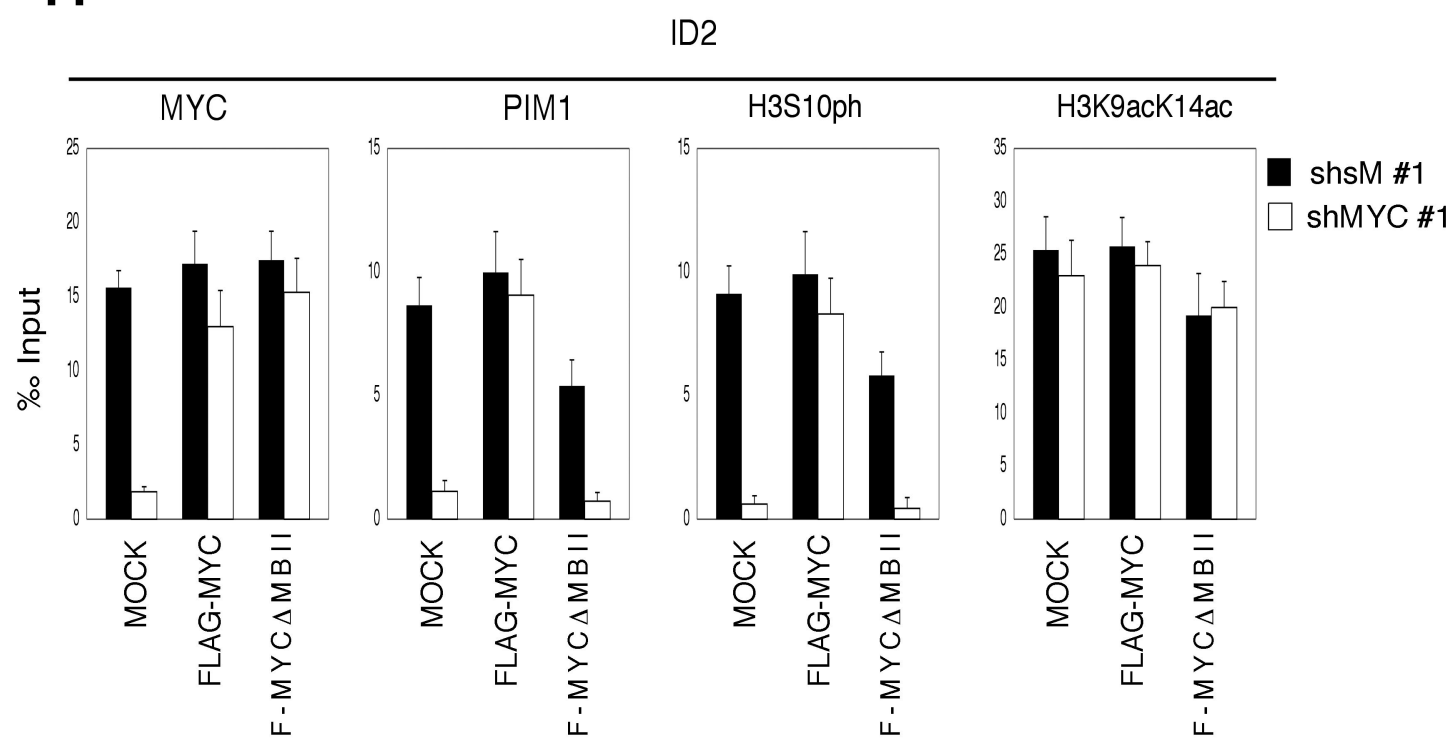

Fig. 6 
a

FOSL1

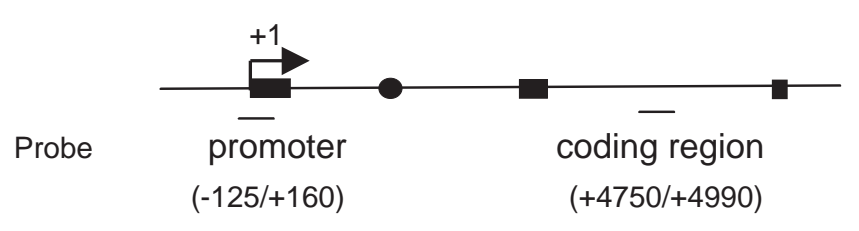

b

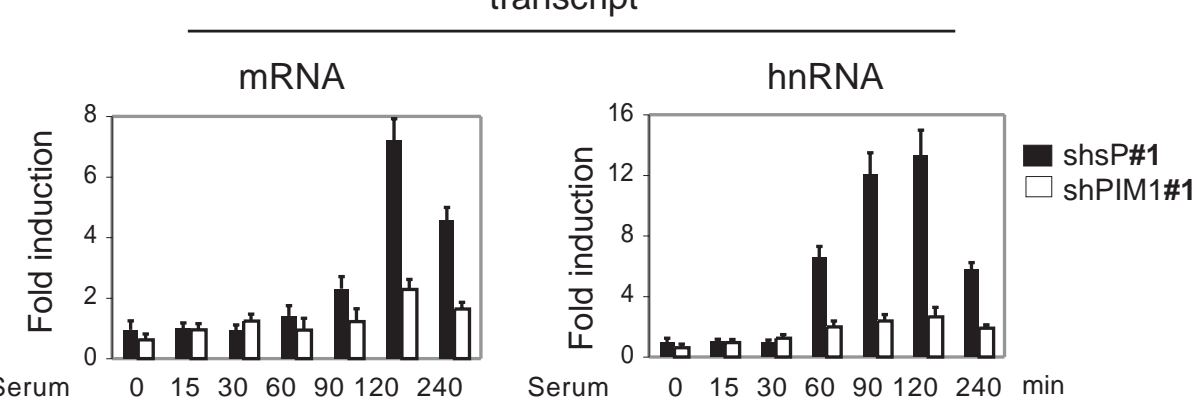

d

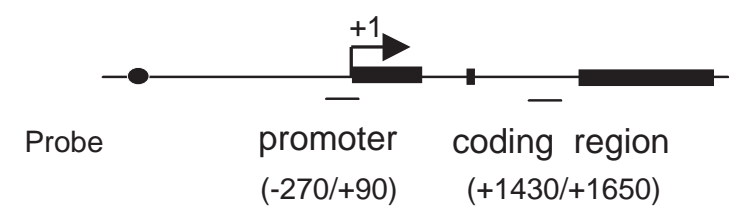

transcript

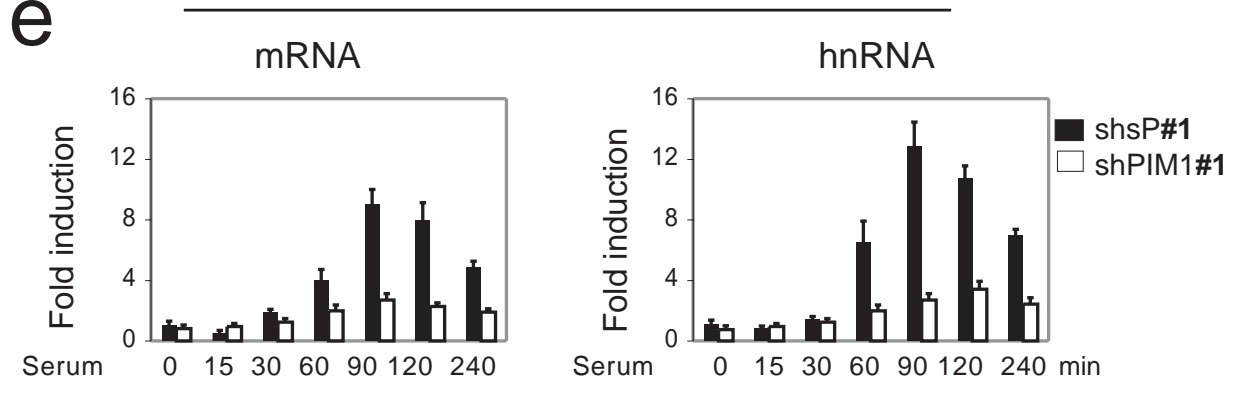

C
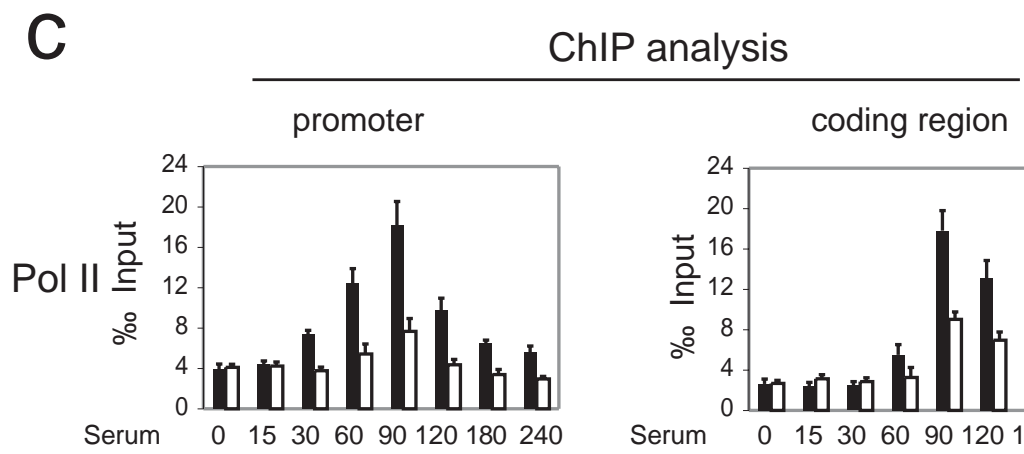

Serum $\quad 0 \quad 15306090120180240$

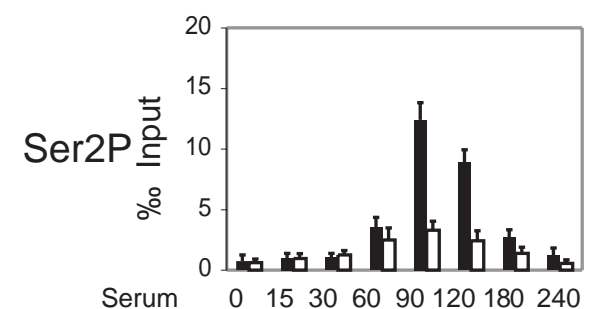

Serum $01530 \quad 6090120180240 \mathrm{~min}$

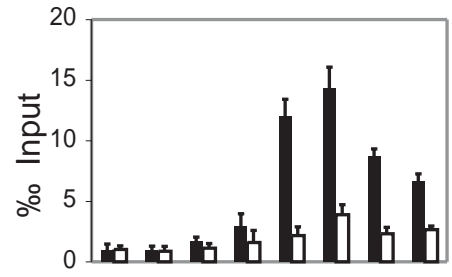

Serum 015306090120180240 min

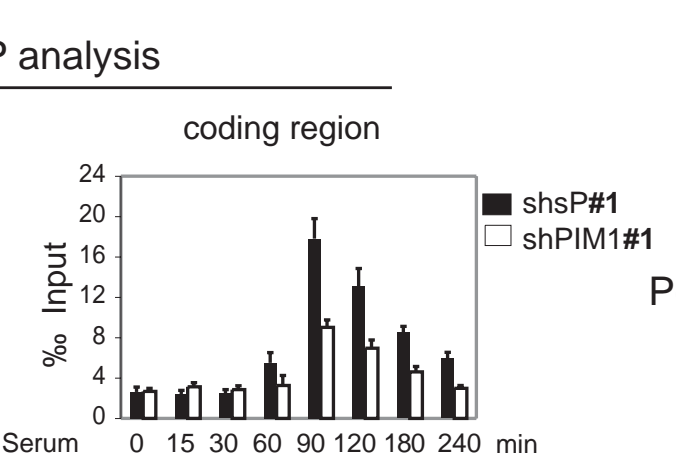

f

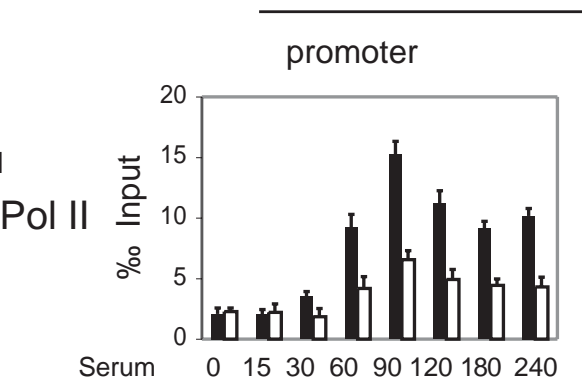

ChIP analysis
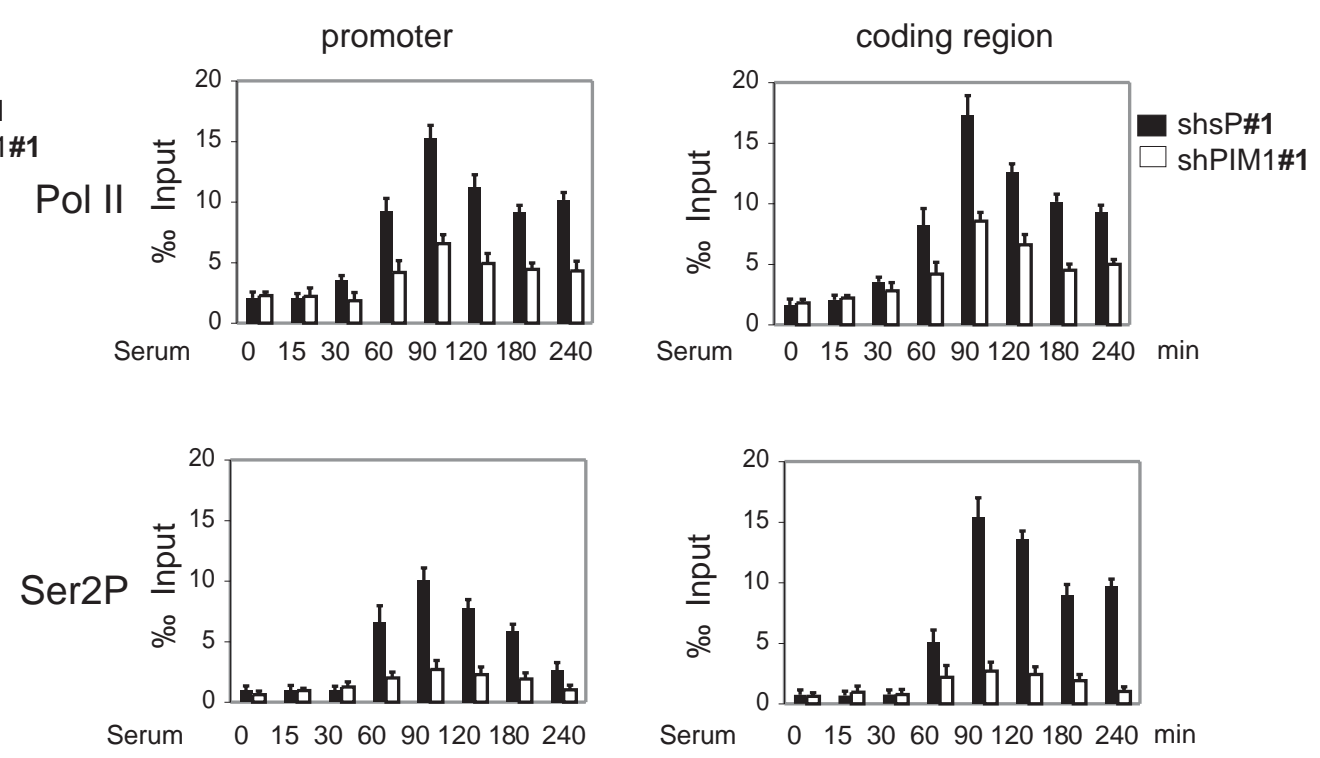

Fig. 7 
a

\begin{tabular}{|c|c|c|c|c|c|c|}
\hline constructs & & $\begin{array}{l}\text { agar } \\
\text { nies }\end{array}$ & SP1 & MAX & PIM1 & MYC \\
\hline mock & 7 & \pm 1 & 1 & $\mathbf{n}$ & 1 & . \\
\hline shsP\#3 + MYC & 75 & \pm 6 & 1 & & $t$ & $\mathbf{E}$ \\
\hline shPIM1\#3 + MYC & 17 & \pm 3 & 1 & & & 8 \\
\hline shsP\#4 + MYC & 73 & \pm 13 & 1 & & 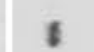 & $\mathbf{I}$ \\
\hline shPIM1\#4 + MYC & 15 & \pm 4 & 1 & $\mathbf{n}$ & & $\mathbf{a}$ \\
\hline mock & 8 & \pm 1 & 1 & $\mathbf{a}$ & 1 & 1 \\
\hline shsM\#3 + PIM1 & 65 & \pm 5 & 1 & & $\mathbf{0}$ & 1 \\
\hline shMYC\#3 + PIM1 & 17 & \pm 4 & i & & $\mathbf{1}$ & \\
\hline shsM\#4 + PIM1 & 54 & \pm 5 & i & 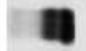 & 0 & $\pi$ \\
\hline shMYC\#4 + PIM1 & 12 & \pm 3 & $i$ & $\mathbf{n}$ & $\mathbf{0}$ & \\
\hline & & & $\begin{array}{l}1 \\
\stackrel{d}{2} \\
\text { 젱 }\end{array}$ & $\begin{array}{l}1 \\
\text { N } \\
\text { 종 }\end{array}$ & $\begin{array}{l}' \\
\omega \\
\text { O } \\
\text { 제 }\end{array}$ & 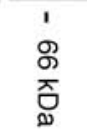 \\
\hline
\end{tabular}

C

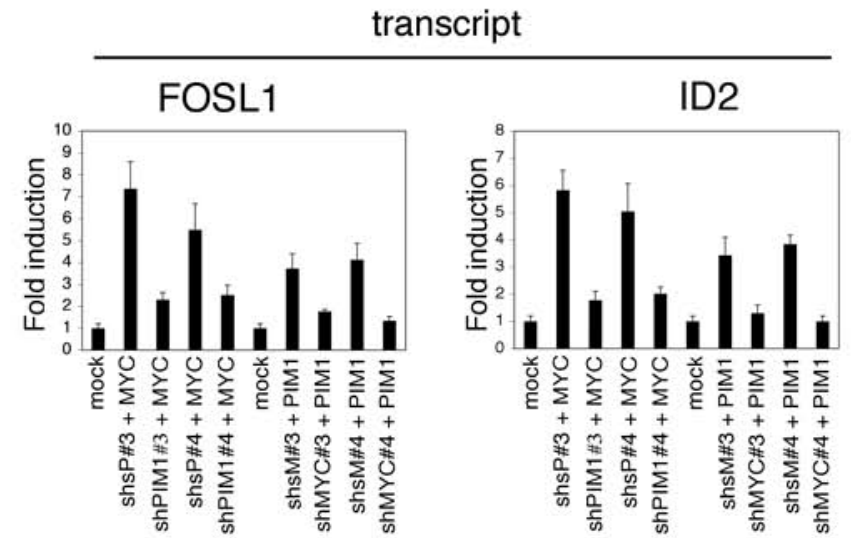

e

ChIP analysis
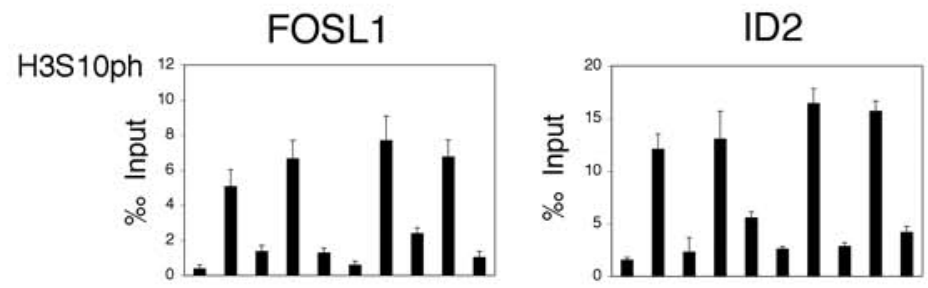

PIM1

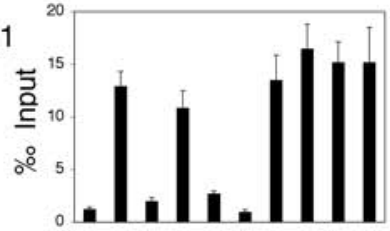

MYC

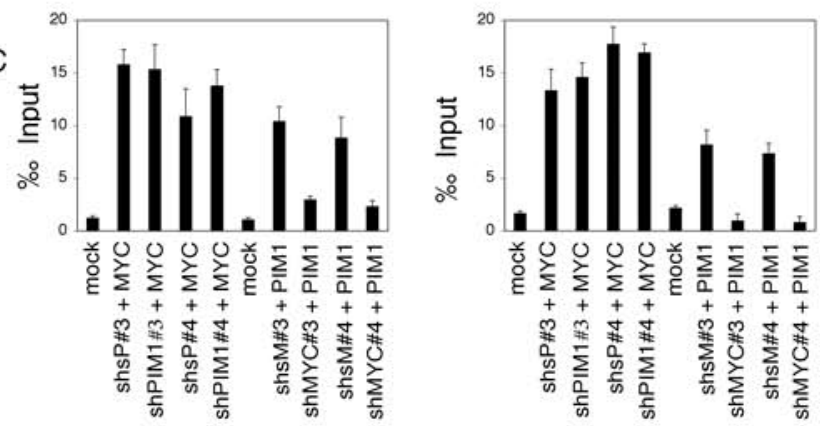

b

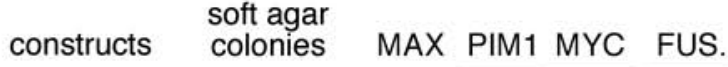

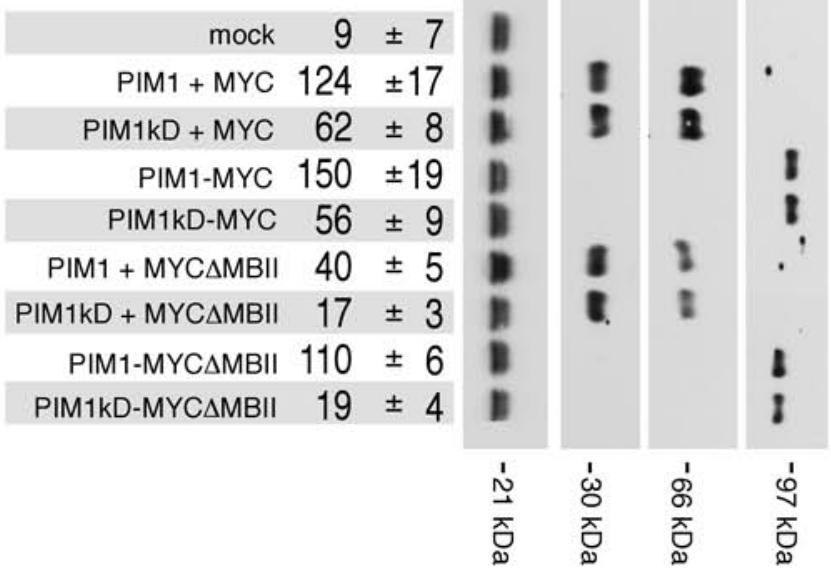

d

\begin{tabular}{ll}
\multicolumn{2}{c}{ transcript } \\
\hline FOSL1 & ID2
\end{tabular}
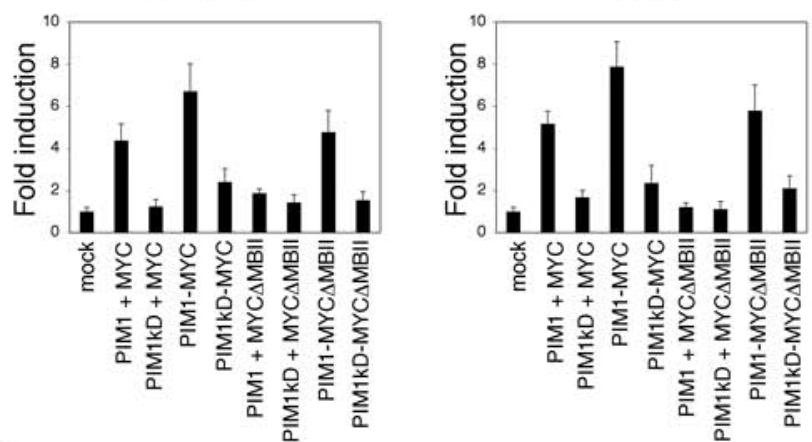

f

ChIP analysis
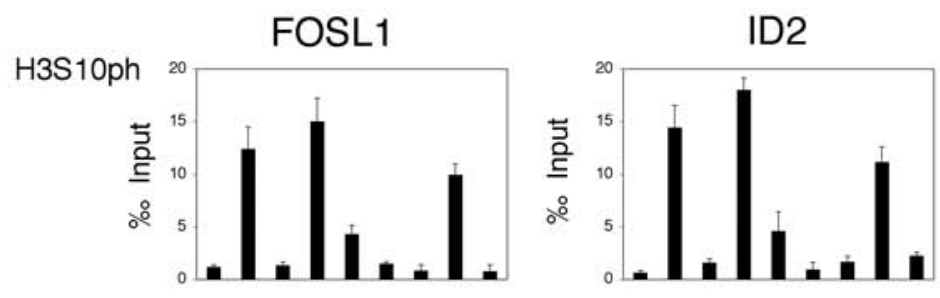

PIM1
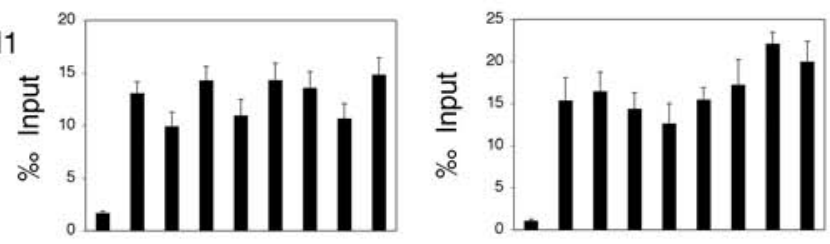

MYC

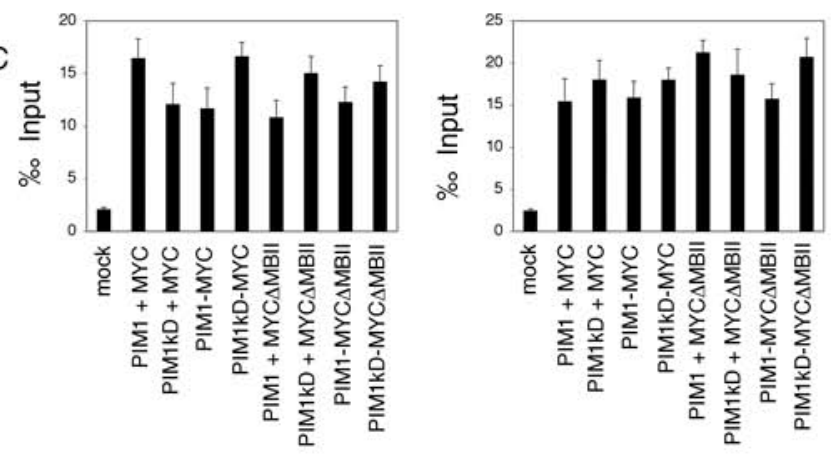

